University of Windsor

Scholarship at UWindsor

$12-3-2010$

\title{
Intramolecular Nicholas Reactions in the Synthesis of Dibenzocycloheptanes. Synthesis of Allocolchicine NSC 51046 and Analogues and the Formal Synthesis of (-)-Allocolchicine
}

\author{
Sinisa Djurdjevic \\ University of Windsor \\ Fei Yang \\ University of Windsor \\ James R. Green \\ University of Windsor
}

Follow this and additional works at: https://scholar.uwindsor.ca/chemistrybiochemistrypub

Part of the Biochemistry, Biophysics, and Structural Biology Commons, and the Chemistry Commons

\section{Recommended Citation}

Djurdjevic, Sinisa; Yang, Fei; and Green, James R.. (2010). Intramolecular Nicholas Reactions in the Synthesis of Dibenzocycloheptanes. Synthesis of Allocolchicine NSC 51046 and Analogues and the Formal Synthesis of (-)-Allocolchicine. Journal of Organic Chemistry, 75 (23), 8241-8251.

https://scholar.uwindsor.ca/chemistrybiochemistrypub/76

This Article is brought to you for free and open access by the Department of Chemistry and Biochemistry at Scholarship at UWindsor. It has been accepted for inclusion in Chemistry and Biochemistry Publications by an authorized administrator of Scholarship at UWindsor. For more information, please contact scholarship@uwindsor.ca. 


\section{Intramolecular Nicholas reactions in the synthesis of}

\section{dibenzocycloheptanes. The synthesis of allocolchicine}

NSC 51046 and analogs, and the formal synthesis of (-)-

\section{allocolchicine.}

Sinisa Djurdjevic, ${ }^{, *}$ Fei Yang, ${ }^{\dagger}$ and James R. Green* ${ }^{\dagger}$

'Department of Chemistry and Biochemistry, University of Windsor, Windsor, ON, N9B 3P4, Canada, and ${ }^{*}$ St. Clair College of Applied Arts and Technology, Windsor, ON, N9A 6S4, Canada.

jgreen@uwindsor.ca

RECEIVED DATE (to be automatically inserted after your manuscript is accepted if required according to the journal that you are submitting your paper to)

Tel: (519)-253-3000, Ext. 3545. Fax: (519)-973-7098.<smiles>[R]c1ccc2c(c1[R])C#CCCc1c([R6])c([R5])c([R5])c([R5])c1-2</smiles><smiles>[R]c1ccc2c(c1[R])[C@@H](NC(C)=O)CCc1c([R6])c([R5])c([R])c([R5])c1-2</smiles> 
The preparation of dibenzocycloheptyne- $\mathrm{Co}_{2}(\mathrm{CO})_{6}$ complexes by intramolecular Nicholas reactions of biaryl-2-propargyl alcohol- $\mathrm{Co}_{2}(\mathrm{CO})_{6}$ derivatives is described. Reductive decomplexation of the dibenzocycloheptyne- $\mathrm{Co}_{2}(\mathrm{CO})_{6}$ complexes affords the corresponding dibenzocycloheptenes, individual members of which have been employed in a formal total synthesis of (-)-allocolchicine, the preparation of 6,7-dihydro-3,4,9,10,11-pentamethoxy-5H-dibenzo[a,c]cyclohepten-5-one, and the enantioselective total syntheses of NSC 51046 and its 3,8,9,10-tetramethoxy regioisomer.

\section{Introduction}

The allocolchicines are a group of compounds containing a tricyclic 6,7,6-system with a highly oxygen substituted A ring. Individual members of this group, including (-)-allocolchcine (1), Nacetylcolchicinol methyl ether (NSC 51046, 2), N-acetylcolchicinol (3) and its dihydrogenphosphate (ZD 6126, 4) have gained considerable attention by virtue of having been found to be active against a number of cancer cell lines. ${ }^{1}$ These act by inhibiting tubulin assembly and polymerization, therefore arresting cell mitosis. A number of additional naturally occurring allocolchicines, including (-)androbiphenyline (4), (-)-colchibiphenyline (5), (-)-jerusalemine (6), (-)-salimine (7), and (-)suhailamine have also been isolated $;^{2}$ the latter two of these have undergone structural revision or are structurally in question. ${ }^{3}$ 


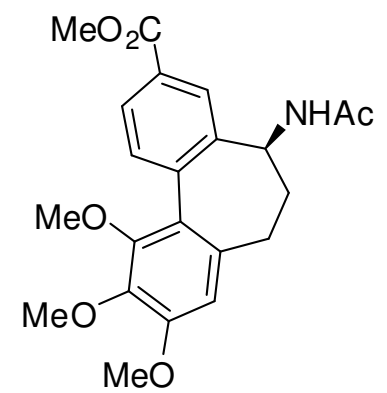

1

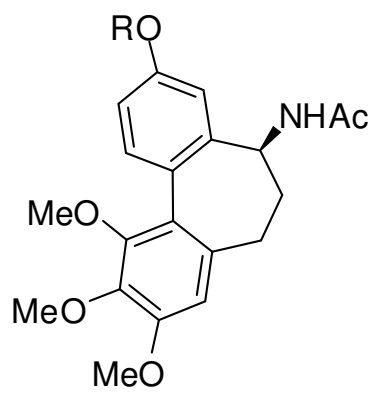

2, $\mathrm{R}=\mathrm{Me}$

3, $\mathrm{R}=\mathrm{H}$

4, $\mathrm{R}=\mathrm{P}(\mathrm{O})(\mathrm{OH})_{2}$<smiles>COc1cc2c(c(OC)c1OC)-c1ccc(O)c(O)c1[C@H](NC(C)=O)CC2</smiles>

4, $\mathrm{R}=\mathrm{Me}$ $5, \mathrm{R}=\mathrm{H}$

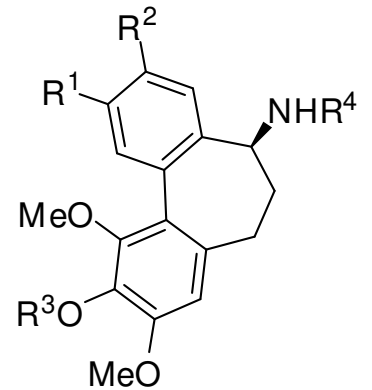

6, $\mathrm{R}^{1}=\mathrm{OH}, \mathrm{R}^{2}=\mathrm{OMe}$ $\mathrm{R}^{3}=\mathrm{H}, \mathrm{R}^{4}=\mathrm{Me}$, 7, $\mathrm{R}^{1}=\mathrm{CO}_{2} \mathrm{Me}, \mathrm{R}^{2}=\mathrm{OH}$ $R^{3}=M e, R^{4}=A c$

Figure 1. Common allocolchicines.

Synthetic access to the allocolchicines historically has been based on oxidation of colchicine itself, ${ }^{4}$ although racemic syntheses or those involving resolution are known. ${ }^{3,5}$ More recently, the activity of these compounds has stimulated an interest in the enantioselective synthesis of allocolchicines. Initiated by Wulff's Diels-Alder based synthesis of (-)-allocolchicine itself, ${ }^{6}$ members of this class of compounds have seen synthesis by way of enyne metathesis/Diels-Alder reactions ((-)-N-acetylcolchicine analogs), oxidative coupling and copper mediated cross coupling ((-)-N-acetylallocolchicinol),${ }^{8} \mathrm{C}-\mathrm{H}$ activation reactions ((-)-allocolchicine formal synthesis) ${ }^{9}$ and aldol condensation chemistry ((-)-Nacetylallocolchicinol) ${ }^{8 b, 10}$ In addition, a recent siloxane coupling/ring expansion reaction chemistry approach to ( \pm )-NSC 51046 has been reported. ${ }^{11}$

The application of alkyne- $\mathrm{Co}_{2}(\mathrm{CO})_{6}$ complexes in the synthesis of seven- membered ring compounds has been demonstrated by our group ${ }^{12}$ and by other groups,${ }^{13}$ most often based on Nicholas 
reaction chemistry. The reactions are suited to seven membered ring synthesis due to fact that complexation of alkynes to $\mathrm{Co}_{2}(\mathrm{CO})_{6}$ induces a change in bond angle to ca. $140^{\circ},{ }^{14}$ in that $\mathrm{S}_{\mathrm{N}} 1$ ' reactivity on the cationic propargyldicobalt complex to give a 5- membered ring system does not occur, and since the electrophilicity of these cations is such that arenes substituted with electron donating groups are sufficiently nucleophilic for facile reaction. ${ }^{15}$ In particular, given the propensity of propargyldicobalt cations for reaction with electron rich arenes and the demonstrated ability of (Z)-arylalkene substituted propargyl acetate complexes to react to form benzocycloheptyne- $\mathrm{Co}_{2}(\mathrm{CO})_{6}$ complexes, ${ }^{12 \mathrm{~d}}$ we considered the potential applicability of intramolecular Nicholas reaction chemistry to dibenzocycloheptanes and consequently allocolchicines to be highly promising. We have reported on the viability of this approach in preliminary form, and now describe this chemistry in complete fashion. ${ }^{16}$

\section{Results and Discussion}

The general outline of the access to the 6-7-6 system was envisioned to occur by construction of the $\mathrm{Co}_{2}(\mathrm{CO})_{6}$ complexes of biaryl-2-propargyl alcohol derivatives (8), which would deliver dibenzocycloheptyne- $\mathrm{CO}_{2}(\mathrm{CO})_{6}$ complexes (9) in the presence of a Lewis acid. The former were prepared in most instances by Suzuki-Miyaura coupling reactions of arylboronic acids (10) with bromobenzaldehydes (11) according the conditions employed by Fürstner, ${ }^{17}$ giving biaryl-2carboxaldehydes (12a-f) in good yield (Scheme 1, Table 1). These aldehydes were subjected to the Corey-Fuchs protocol, with trapping of the resulting acetylide by paraformaldehyde (Scheme 2). The resultant propargyl alcohols (13a-f) were formed in fair to good yields (56-80\%) except in the case of thienyl substituted 13d (40\% yield); in this case a significant amount of carbene insertion product 14 (53\%) was formed competitively. Acetylation of 13a-f under standard conditions and complexation with $\mathrm{Co}_{2}(\mathrm{CO})_{8}$ then afforded 8a-f in good to excellent yields.

Scheme 1. Suzuki-Miyaura coupling reactions. 


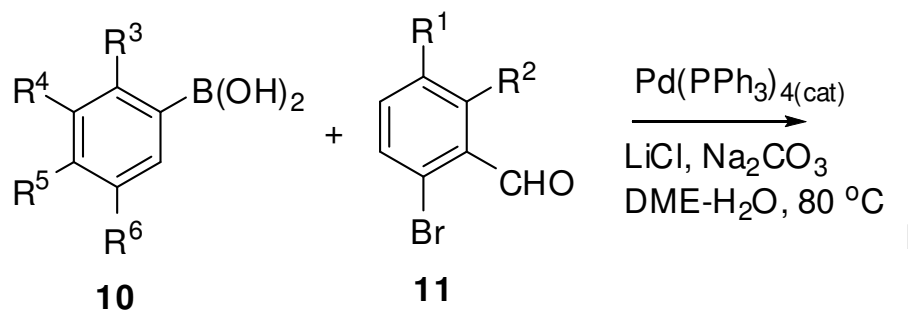<smiles>[R7]c1ccc(-c2cc([P])c(P)c([R])c2[R])c(C=O)c1[R]</smiles><smiles>COc1ccc(-c2ccsc2)c(C=O)c1</smiles>
$10 R^{3} \quad R^{4} \quad R^{5} \quad R^{6}$
$11 R^{1} \quad R^{2}$
$12 \begin{array}{llllll}R^{1} & R^{2} & R^{3} & R^{4} & R^{5} & R^{6}\end{array}$
a OMe OMe OMe $\mathrm{H}$
a $\mathrm{OMe} \mathrm{H}$
b $\mathrm{H} \quad \mathrm{Me} \quad \mathrm{H} \quad \mathrm{Me}$
b $\mathrm{H} \quad \mathrm{H}$
d $\mathrm{H}$ OMe OMe OMe
c $\mathrm{OMe} \mathrm{OMe}$
a $\mathrm{OMe} \mathrm{H}$ OMe OMe OMe $\mathrm{H}$
b $\mathrm{H} \quad \mathrm{H}$ OMe OMe OMe $\mathrm{H}$
$\begin{array}{llllll}\text { c } \mathrm{OMe} & \mathrm{H} & \mathrm{H} & \mathrm{Me} & \mathrm{H} & \mathrm{Me}\end{array}$
e $\mathrm{OMe} \mathrm{H} \quad \mathrm{H}$ OMe OMe OMe
10c<smiles>COc1ccsc1</smiles>
f OMe OMe OMe OMe OMe $\mathrm{H}$

Scheme 2. Preparation of Biaryl-2-propargyl Acetate- $\mathrm{Co}_{2}(\mathrm{CO})_{6}$ Complexes $(8)$.

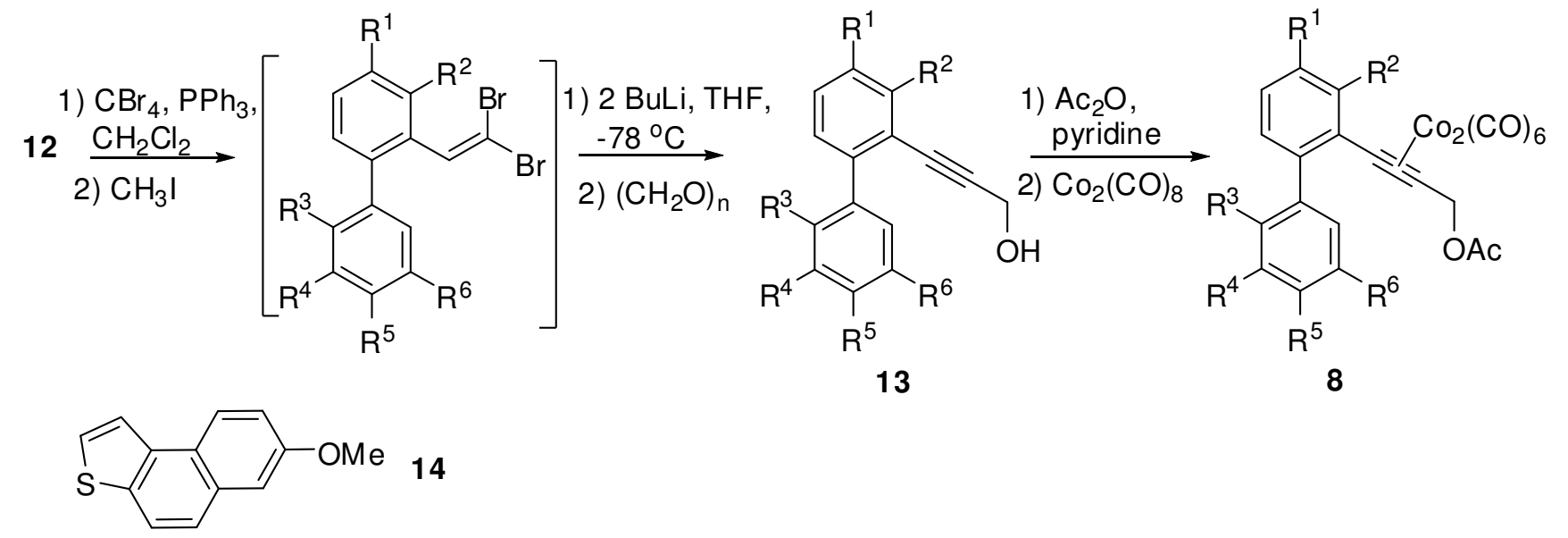

Table 1. Preparation of $\mathbf{8 a - g}$

\begin{tabular}{|l|l|l|l|l|}
\hline 10 & 11 & $12^{\mathrm{a}}$ & 13 & 8 \\
\hline $10 a$ & $11 a$ & $12 a(85)$ & $13 a(80)$ & $8 a(86)$ \\
\hline $10 a$ & $11 b$ & $12 b(92)$ & $13 b(78)$ & $8 b(84)$ \\
\hline $10 b$ & $11 a$ & $12 c(79)$ & $13 c(57)$ & $8 c(91)$ \\
\hline $10 c$ & $11 a$ & $12 d(81)$ & $13 d(40)$ & $8 d(77)$ \\
\hline
\end{tabular}




\begin{tabular}{|l|l|l|l|l|}
\hline 10d & 11a & 12e (76) & 13e (61) & 8e (84) \\
\hline 10a & 11c & 12f (81) & 13f (56) & 8f (98) \\
\hline
\end{tabular}

${ }^{a}$ Yields are in parentheses.

Two additional substrates were prepared by procedures other than the standard one. Unsubstituted $\mathbf{8 g}$ was obtained by way of Sonogashira reaction between 2-iodobiphenyl and propargyl alcohol to give $\mathbf{1 3 g}$ (87\% yield) (Scheme 3), which was in turn subjected to acetylation and complexation with $\mathrm{Co}_{2}(\mathrm{CO})_{8}$ under conditions analogous to 13a-f, affording $\mathbf{8 g}$ in $86 \%$ yield.

Scheme 3. Formation of $8 g$.



1) $\mathrm{Ac}_{2} \mathrm{O}$, pyridine 2) $\mathrm{Co}_{2}(\mathrm{CO})_{8}$ $8 \mathrm{~g}$<smiles>CC(=O)OCC#Cc1ccccc1-c1ccccc1</smiles>

Carbomethoxy- substituted $\mathbf{8 h}$, the propargyl ether- $\mathrm{Co}_{2}(\mathrm{CO})_{6}$ complex envisioned as the precursor to allocolchicine itself, required a modified approach for its preparation. In this case, methyl 4-bromo-3-iodobenzoate, prepared by conventional esterification of the corresponding acid, ${ }^{18}$ was subjected to Sonogashira reaction with propargyl methyl ether to afford $\mathbf{1 5}$ in $92 \%$ yield (Scheme 4). The Suzuki-Miyaura reaction of this halide with 2,3,4-trimethoxyboronic acid was somewhat problematic, as convention conditions resulted in predominant boronic acid hydrolysis and recovery of substantial 15, with only a small amount of 16 isolated $\left(27 \%\right.$ yield). Use of $\operatorname{Pd}_{2}(\mathrm{dba})_{3}$ with $\mathrm{PCy}_{3}$, however, gave 16 in acceptable yield (53\%, 77\% based on recovered starting material [brsm]), with recovery of $15(31 \%)$. While most of the material was carried forward using this protocol, it was found 
ultimately that use of the PEPPSI-iPr catalyst enabled formation of $\mathbf{1 6}$ in $\mathbf{7 9 \%}$ yield. ${ }^{19}$ Formation of $\mathbf{8 h}$ was accomplished from 16 in a straightforward manner $(83 \%$ yield $)$ with $\mathrm{Co}_{2}(\mathrm{CO})_{8}$.

Scheme 4. Preparation of $\mathbf{8 h}$.
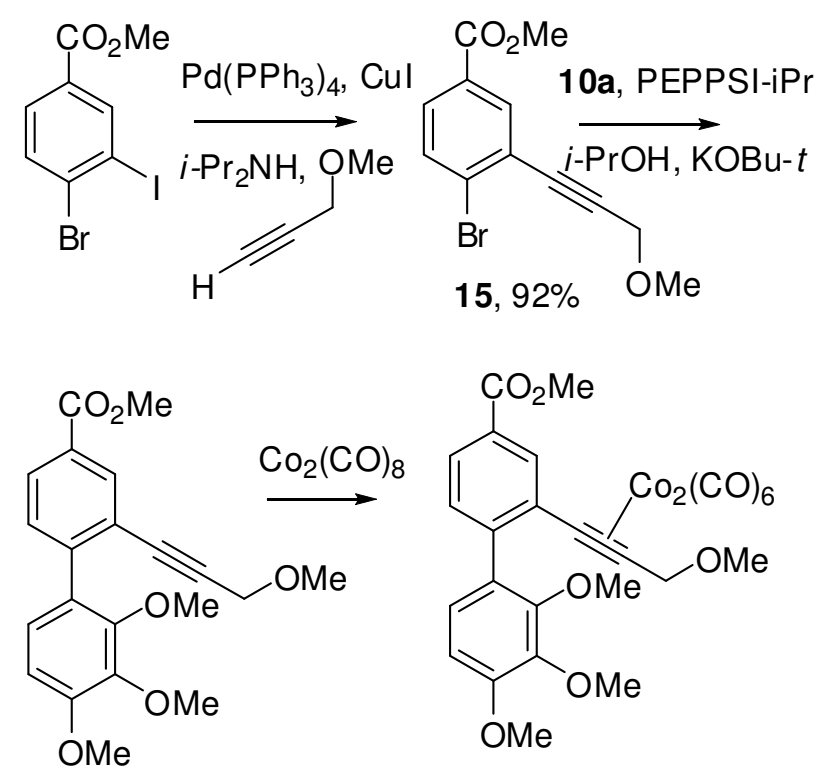

$16,79 \%$

$8 \mathrm{~h}, 83 \%$

\section{PEPPSI-iPr}

$\mathrm{Ar}=2,6$-diisopropylphenyl<smiles></smiles>

With the Nicholas reaction precursors in hand, attention was turned to investigation of the cyclizations. Under conditions developed previously for benzocycloheptyne ring closure reactions, 8a (0.005 $\mathrm{M}$ in $\mathrm{CH}_{2} \mathrm{Cl}_{2}$ ) underwent reaction in the presence of $\mathrm{BF}_{3}-\mathrm{OEt}_{2}$ (3 equiv), giving dibenzocycloheptyne 9a over 2.5 h (56\% yield) (Table 2, entry 1). As a small amount of decomposition was evident chromatographically during this process, and with the belief that this could be due to the acid liberated during the substitution process, the reaction was conducted with the addition of 1.5 equiv. $i$-Pr ${ }_{2}$ NEt. Although the reaction occurred somewhat more slowly (6 h) (entry 2), 9a could be isolated in improved yield $(71 \%)$. These conditions (3 equiv. $\mathrm{BF}_{3}-\mathrm{OEt}_{2}, 1.5$ equiv. $i$ - $\mathrm{Pr}_{2} \mathrm{NEt}, 0.005-0.01 \mathrm{M}$ ) were applied to $\mathbf{8 b - 8 h}$ (Table 2), and afforded fair to excellent yields of $\mathbf{9 b - h}$. While there was some variation in required reaction time $(4.5-16 \mathrm{~h})$ and substrate, there was no particular correlation between reaction 
time and substitution pattern of the arene behaving as nucleophile. In the case of trimethoxy substituted $\mathbf{8 b}$, TLC analysis suggested the onset of some decomposition without complete conversion at $16 \mathrm{~h}$, so that the reaction was terminated at this point and small amount of $\mathbf{8 b}(10 \%)$ could be recovered in addition to the isolated $\mathbf{9 b}$ (59\% yield) (entry 3). 3-Thienyl substituted case $\mathbf{8 d}$ underwent competitive cyclization at C-2' and C-4', affording 9d and 9d' and a regioisomeric mixture (82\%, 45:55 9d:9d') (entry 5). It is also worthy of note that $\mathbf{8 c} \cdot \mathbf{9 c}($ entry 4) and $\mathbf{8 g} \bullet \mathbf{9 g}$ (entry 8) proceeded uneventfully, as the less electron rich arene nucleophiles would be of borderline reactivity and insufficient reactivity, respectively, for participation in intermolecular Nicholas reactions. ${ }^{15}$ Evidence of restricted rotation about the aryl-aryl bond was present for several of the cyclization products, as all dibenzocycloheptyne$\mathrm{Co}_{2}(\mathrm{CO})_{6}$ complexes bearing an additional substituent ortho to the biaryl gave diastereotopic $\mathrm{CH}_{2}$ 's for the propargylic hydrogen atoms in the ${ }^{1} \mathrm{H}$ NMR spectra $(\mathbf{9 a}, \mathbf{b}, \mathbf{f}, \mathbf{g})$. In addition, those bearing substitutents ortho to the cycloheptyne either gave a diastereotopic $\mathrm{CH}_{2}(\mathbf{9 c})$ or one right at coalescence (9h).

Table 2. Intramolecular Nicholas Reactions of $\mathbf{8 .}$ 


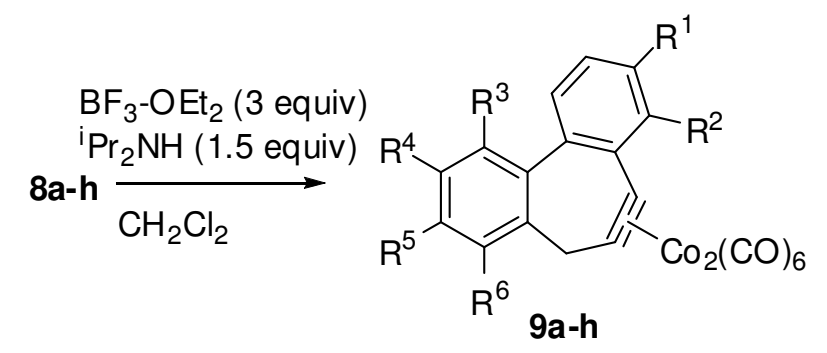

$\begin{array}{ccccccc}\mathbf{9} & \mathrm{R}^{1} & \mathrm{R}^{2} & \mathrm{R}^{3} & \mathrm{R}^{4} & \mathrm{R}^{5} & \mathrm{R}^{6} \\ \text { a } & \mathrm{OMe} & \mathrm{H} & \mathrm{OMe} & \mathrm{OMe} & \mathrm{OMe} & \mathrm{H} \\ \mathbf{b} & \mathrm{H} & \mathrm{H} & \mathrm{OMe} & \mathrm{OMe} & \mathrm{OMe} & \mathrm{H} \\ \mathbf{c} & \mathrm{OMe} & \mathrm{H} & \mathrm{H} & \mathrm{Me} & \mathrm{H} & \mathrm{Me} \\ \mathbf{e} & \mathrm{OMe} & \mathrm{H} & \mathrm{H} & \mathrm{OMe} & \mathrm{OMe} & \mathrm{OMe} \\ \mathbf{f} & \mathrm{OMe} & \mathrm{OMe} & \mathrm{OMe} & \mathrm{OMe} & \mathrm{OMe} & \mathrm{H} \\ \mathbf{g} & \mathrm{H} & \mathrm{H} & \mathrm{H} & \mathrm{H} & \mathrm{H} & \mathrm{H} \\ \mathbf{h} & \mathrm{CO}_{2} \mathrm{Me} & \mathrm{H} & \mathrm{OMe} & \mathrm{OMe} & \mathrm{OMe} & \mathrm{H}\end{array}$<smiles>COc1ccc2c(c1)C=C(C(=O)OCc1ccccc1)Cc1sccc1-2</smiles>

9d

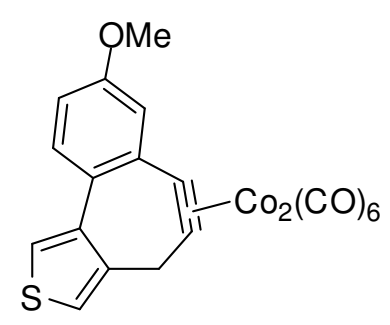

9d'

\begin{tabular}{|l|l|l|l|l|}
\hline Entry & $\mathbf{8}$ & time & $\mathbf{9}$ & Yield (\%) \\
\hline 1 & $\mathbf{8 a}$ & $2.5 \mathrm{~h}$ & $\mathbf{9 a}$ & $56^{a}$ \\
\hline 2 & $\mathbf{8 a}$ & $6 \mathrm{~h}$ & $\mathbf{9 a}$ & 71 \\
\hline 3 & $\mathbf{8 b}$ & $16 \mathrm{~h}$ & $\mathbf{9 b}$ & $59(66)^{b}$ \\
\hline 4 & $\mathbf{8 c}$ & $6 \mathrm{~h}$ & $\mathbf{9 c}$ & 85 \\
\hline 5 & $\mathbf{8 d}$ & $5 \mathrm{~h}$ & $\mathbf{9 d}$ & $82^{c}$ \\
\hline 6 & $\mathbf{8 e}$ & $4.5 \mathrm{~h}$ & $\mathbf{9 e}$ & 91 \\
\hline 7 & $\mathbf{8 f}$ & $4.5 \mathrm{~h}$ & $\mathbf{9 f}$ & 83 \\
\hline 8 & $\mathbf{8 g}$ & $16 \mathrm{~h}$ & $\mathbf{9 g}$ & 58 \\
\hline 9 & $\mathbf{8 h}$ & $5 \mathrm{~h}$ & $\mathbf{9 h}$ & 84 \\
\hline
\end{tabular}

${ }^{a}$ No $i$-Pr ${ }_{2}$ NEt added. ${ }^{b}$ Yields in parentheses based on recovered starting material. ${ }^{c}$ Isolated as a 45:55 9d:9d' mixture. 
Removal of the $\mathrm{Co}_{2}(\mathrm{CO})_{6}$ fragment for use in synthesis require concomitant conversion of the alkyne function into one compatible with the seven- membered ring. ${ }^{20,21}$ The most commonly employed reagent for this purpose, $\mathrm{Bu}_{3} \mathrm{SnH}$, has caused some isomerization in related benzocycloheptyne cases; ${ }^{12 \mathrm{c}}$ consequently we chose to apply a modification of Isobe's hydrosilylation protocol ${ }^{20}$ that would afford the alkene. Addition of triethylsilane to the dibenzocycloheptyne complexes 9 in the presence of bis(trimethylsilylacetylene) (BTMSE) gave a regioisomeric mixture of silylated cycloheptenes, which were not isolated, but subjected to in situ desilylation with trifluoroacetic acid (TFA) to give the dibenzocycloheptenes 17. These dibenzocycloheptenes $(\mathbf{1 7 a}, \mathbf{e}, \mathbf{f}, \mathbf{h})$ were isolated in good to excellent yields, and with no evidence of double bond isomerization during the reductive decomplexation process (Table 3).

Table 3. Conversion of $\mathbf{9}$ to dibenzocycloheptanones $\mathbf{1 8 .}$

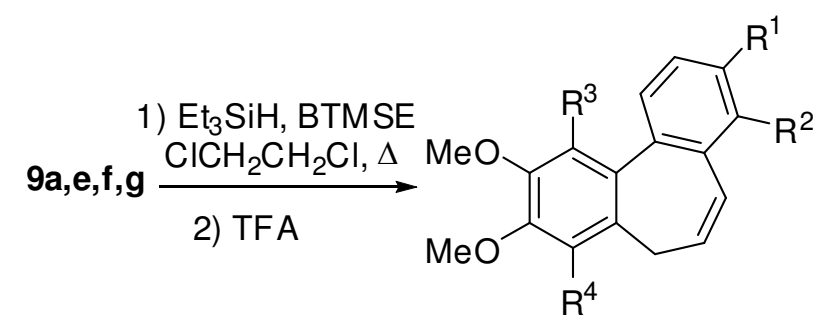

$17 a, e, f, g$

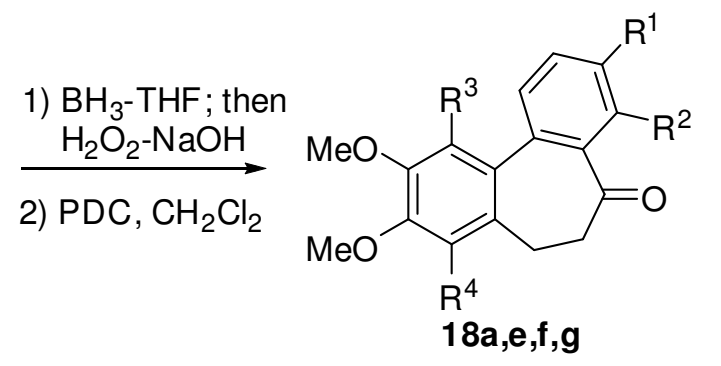

17a, 18a $R^{1}=O M e, R^{2}=H, R^{3}=O M e, R^{4}=H$

17e, 18e $R^{1}=O M e, R^{2}=H, R^{3}=H, R^{4}=O M e$

17f, $18 f R^{1}=$ OMe, $R^{2}=$ OMe, $R^{3}=O M e, R^{4}=H$

17g, 18g, $R^{1}=\mathrm{CO}_{2} \mathrm{Me}, \mathrm{R}^{2}=\mathrm{H}, \mathrm{R}^{3}=\mathrm{OMe}, \mathrm{R}^{4}=\mathrm{H}$

\begin{tabular}{|l|l|c|c|c|}
\hline $\mathbf{9}$ & $\mathbf{1 7}$ & Yield (\%) & $\mathbf{1 8}$ & Yield (\%) \\
\hline $\mathbf{9 a}$ & $\mathbf{1 7 a}$ & 97 & $\mathbf{1 8 a}$ & $80^{\mathrm{a}}$ \\
\hline $\mathbf{9 e}$ & $\mathbf{1 7 e}$ & 94 & $\mathbf{1 8 e}$ & 68 \\
\hline
\end{tabular}




\begin{tabular}{|c|c|c|c|c|}
\hline $\mathbf{9 f}$ & $\mathbf{1 7 f}$ & 90 & $\mathbf{1 8 f}$ & 67 \\
\hline $\mathbf{9 h}$ & $\mathbf{1 7 h}$ & 79 & $\mathbf{1 8 h}$ & 81 \\
\hline
\end{tabular}

${ }^{a}$ Oxidation under Swern conditions

(oxalyl chloride-DMSO, $\mathrm{Et}_{3} \mathrm{~N}$ ).

Formal synthesis of (-)-allocolchicine. Since several of the existing syntheses of allocolchicines employ the dibenzocycloheptanones (18) as critical intermediates, conversion of dibenzocycloheptenes 17 to 18 was considered to be the most prudent approach toward their synthesis. Hydroborationoxidation of $\mathbf{1 7 h}$ with $\mathrm{BH}_{3}-\mathrm{THF} / \mathrm{H}_{2} \mathrm{O}_{2}$, with further oxidation of the intermediate alcohol using PDC, afforded ketone $\mathbf{1 8 h}$ in good yield (81\%). Dibenzocycloheptanone $\mathbf{1 8 h}$ has been converted to (-)allocolchine by Wulff, ${ }^{6}$ and as such this represents a formal synthesis of this natural product.

Similarly, treatment of $\mathbf{1 7 f}$ by $\mathrm{BH}_{3}-\mathrm{THF} / \mathrm{H}_{2} \mathrm{O}_{2}$ followed by oxidation with PDC afforded $\mathbf{1 8 f}(67 \%$ yield). Dibenzocycloheptanone $\mathbf{1 8 f}$ is a degradation product of (-)-androbiphenyline that has been prepared previously by Seitz, ${ }^{22}$ and found to be equally potent to (-)-androbiphenyline in inhibition of tubulin assembly.

Synthesis of (-)-N-acetylcolchicine O-Methyl Ether (NSC 51046) and analog. Conversion of 17a to ketone 18a was accomplished by hydroboration-oxidation with further oxidation under Swern conditions (DMSO-oxalyl chloride, $\mathrm{Et}_{3} \mathrm{~N}$ ), giving the ketone in $80 \%$ yield. Adopting the approach of Wulff's group towards (-)-allocolchicine, the reduction of ketone $\mathbf{1 8 a}$ using the $\mathrm{LiBH}_{4} /$ tartrate derived boronate ester (TARB-NO$)_{2}$ ) protocol of Singaram, ${ }^{23}$ gave alcohol 19a in excellent yield and enantioselectivity (96\% yield, 95\% ee) (Scheme 5). Substitution of azide for the alcohol function by way of zinc azide/diisoproyl azodicarboxylate (DIAD) ${ }^{24}$ afforded 20a $(64 \%)$, while azide reduction $\left(\mathrm{H}_{2}\right.$, Lindlar catalyst) of 20a and acetylation gave NSC 51046 (2) in $88 \%$ (93\% ee), which was spectroscopically identical to literature report. ${ }^{11}$ Recrystallization of $\mathbf{2}$ gave this compound in $>99 \%$ ee. To our knowledge this is the first asymmetric synthesis of 2.

Scheme 5. Completion of Enantioselective Synthesis of NSC 51046 and its 3,8,9,10-tetramethoxy isomer. 


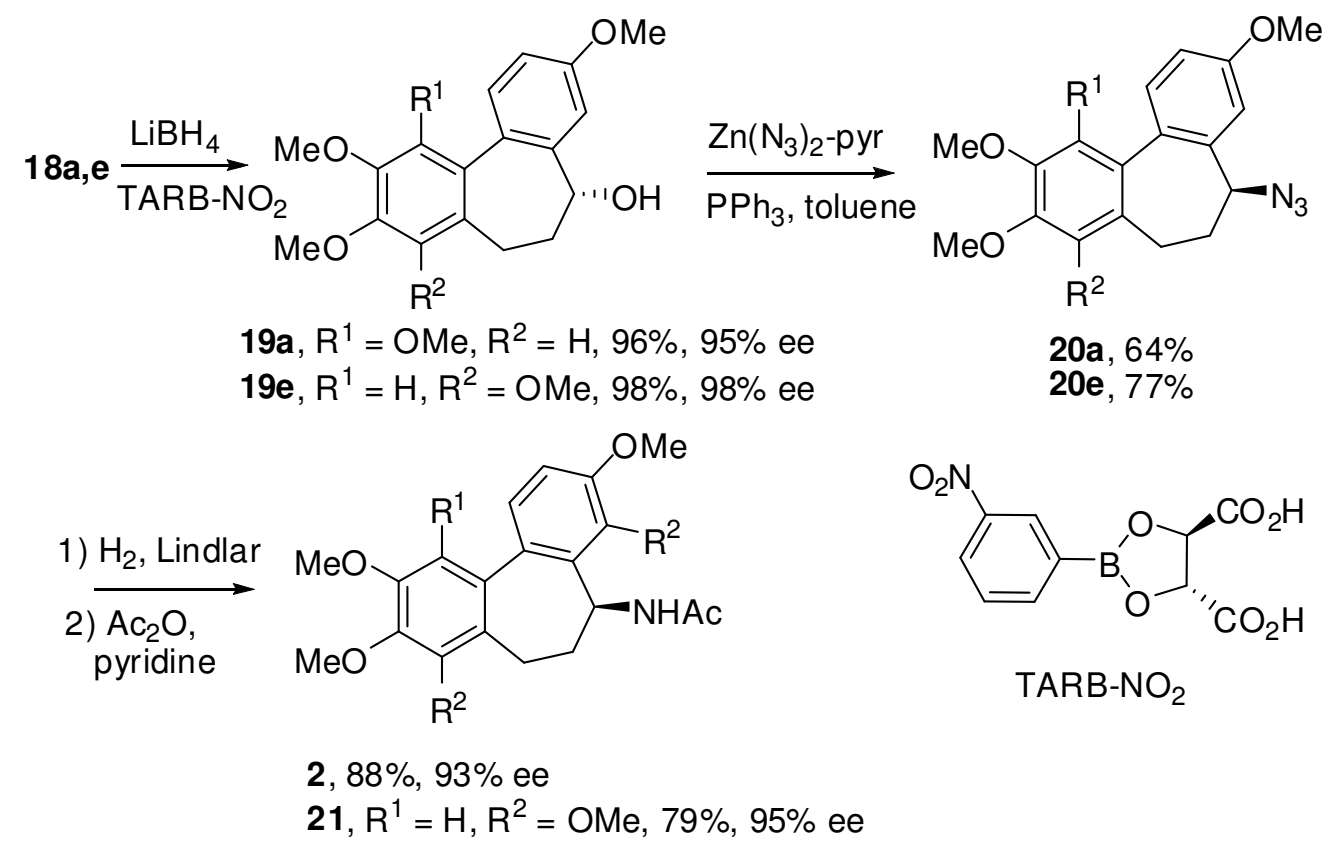

Similarly, dibenzocycloheptene $\mathbf{1 7 e}$ was converted to an isomeric $3,8,9,10-$ tetramethoxyallocolchicine (21). Hydroboration-oxidation of 17e and further oxidation with PDC afforded dibenzocycloheptanone $18 \mathrm{e}$ in $68 \%$ yield. $\mathrm{LiBH}_{4} / \mathrm{TARB}-\mathrm{NO}_{2}$ based reduction afforded the highly enantiomerically enriched alcohol 19e (98\% yield, 98\% ee) provided an extended period substrate/TARB- $\mathrm{NO}_{2}$ mixing and slow $\mathrm{LiBH}_{4}$ addition protocol was followed. ${ }^{25}$ Zinc azide based substitution of the alcohol afforded 20e without incident (77\% yield), while reduction and acetylation gave 21 in 79\% yield (95\% ee); once again, a single recrystallization enriched this compound to $>99 \%$ ee. To our knowledge, this is the first example of an allocolchicine with an 8,9,10- oxygenated A ring.

In summary, intramolecular Nicholas reactions have proven to be effective in the synthesis of dibenzocycloheptyne- $\mathrm{Co}_{2}(\mathrm{CO})_{6}$ complexes. Ready decomplexation to the dibenzocycloheptenes allows application of this methodology to the synthesis of allocolchicines or their derivatives, including tubulin-inhibiting ketone 6,7-dihydro-3,4,9,10,11-pentamethoxy-5H-Dibenzo[a,c]cyclohepten-5-one (18f), a formal total synthesis of (-)-allocolchicine (1), the enantioselective total synthesis of $\mathrm{N}$ acetylallocolchinol O-methyl ether (NSC 51046, 2), and of the 3,8,9,10- tetramethoxy isomer of NSC $51046(\mathbf{2 1})$.

\section{Experimental Section}


General Methods: All reaction solvents were used after passage through a solvent purification system. Commercial $\mathrm{BF}_{3}-\mathrm{OEt}_{2}$ was distilled and stored under nitrogen. All reactions were conducted under a nitrogen atmosphere unless otherwise noted. Flash chromatography was performed as described by Still using silica gel 60 (230-400 mesh). ${ }^{26}$ 2-Bromo-5-methoxybenzaldehyde ${ }^{27}$ and 6-bromo-2,3dimethoxybenzaldehyde ${ }^{28}$ were prepared by literature methods and are $>95 \%$ purity as determined by ${ }^{1} \mathrm{H}$ and ${ }^{13} \mathrm{C}$ NMR spectroscopy. All new compounds are $>95 \%$ purity as determined by ${ }^{1} \mathrm{H}$ and ${ }^{13} \mathrm{C}$ NMR spectroscopy. NMR spectra were run at $500 \mathrm{MHz}$ or $300 \mathrm{MHz}$ for ${ }^{1} \mathrm{H}$ and $125 \mathrm{MHz}$ or $75 \mathrm{MHz}$ for ${ }^{13} \mathrm{C}$ in $\mathrm{CDCl}_{3}$; chemical shifts are given in ppm and coupling constants $(J)$ are given in Hz. High resolution mass spectra were run by time of flight mass spectroscopy, in EI mode, at $70 \mathrm{eV}$.

2',3',4,4'-Tetramethoxy[1,1'-Biphenyl]-2-carboxaldehyde (12a). Prepared according to the method of Fürstner, ${ }^{17} 11 \mathbf{a}(0.3517 \mathrm{~g}, 1.16 \mathrm{mmol})$, afforded 7a $(0.4202,85 \%)$; mp 102-3 ${ }^{\circ} \mathrm{C}$ (hexanes); lit. ${ }^{17} 102-$ $3{ }^{\circ} \mathrm{C}$. This compound $>95 \%$ purity as determined by ${ }^{1} \mathrm{H}$ and ${ }^{13} \mathrm{C}$ NMR spectroscopy.

2',3',4'-Trimethoxy[1,1'-Biphenyl]-2-carboxaldehyde (12b). Prepared according to the method of Fürstner, ${ }^{17} \mathbf{1 1 b}(0.2278 \mathrm{~g}, 1.23 \mathrm{mmol})$, affording 12b (0.3706 g, 92\%); mp 105-5.5 ${ }^{\circ} \mathrm{C}$ (hexanes); lit. ${ }^{17}$ 98-99 ${ }^{\circ} \mathrm{C}$. This compound $>95 \%$ purity as determined by ${ }^{1} \mathrm{H}$ and ${ }^{13} \mathrm{C}$ NMR spectroscopy.

4-Methoxy-3',5'-dimethyl[1,1'-biphenyl]-2-carboxaldehyde (12c). Prepared as adapted from the method of Fürstner, ${ }^{17}$ employing $11 \mathbf{a}(0.9593 \mathrm{~g}, 4.46 \mathrm{mmol})$ and (3,5-dimethylphenyl)boronic acid (6b) $(1.3423 \mathrm{~g}, 8.96 \mathrm{mmol})$ to give $12 \mathrm{c}(0.8472 \mathrm{~g}, 79 \%$ yield $)$, following flash chromatographic purification (15:1 petroleum ether : $\left.\mathrm{Et}_{2} \mathrm{O}\right)$, as a colorless viscous oil which solidified upon standing; $\mathrm{mp} 71-2{ }^{\circ} \mathrm{C}$; IR $(\mathrm{KBr})_{\max } 3006,2917,1688,1604 \mathrm{~cm}^{-1} ;{ }^{1} \mathrm{H}$ NMR $9.97(\mathrm{~s}, 1 \mathrm{H}), 7.52(\mathrm{~d}, \mathrm{~J}=2.8,1 \mathrm{H}), 7.37(\mathrm{~d}, \mathrm{~J}=8.5$, 1H), 7.19 (dd, J = 8.5, 2.8, 1H), 7.06 (br s, 1H), 6.97 (br s, 2H), 3.90 (s, 3H), 2.39 (s, 6H); ${ }^{13} \mathrm{C}$ NMR $192.4,159.0,139.4,137.9,137.4,134.5,131.9,129.3,128.1,121.2,109.7,55.5,21.2$; MS m/e 240 $\left(\mathrm{M}^{+}\right) ;$HRMS m/e for $\mathrm{C}_{16} \mathrm{H}_{16} \mathrm{O}_{2}$ calcd. $240.1150\left(\mathrm{M}^{+}\right)$, found 240.1140 .

5-Methoxy-2-(3-thienyl)benzaldehyde (12d). Prepared as adapted from the method of Fürstner, ${ }^{17}$ employing $11 \mathrm{a}(0.2301 \mathrm{~g}, 1.07 \mathrm{mmol})$ and 3-thienylboronic acid (10c) $(0.2003 \mathrm{~g}, 1.56 \mathrm{mmol})$ to give 12d (0.1900 g, 81\% yield) following flash chromatographic purification (15:1 petroleum ether : $\left.\mathrm{Et}_{2} \mathrm{O}\right)$, 
$\operatorname{mp} 68-69{ }^{\circ} \mathrm{C}\left(\mathrm{CH}_{2} \mathrm{Cl}_{2}\right)$; IR (KBr) ${ }_{\max } 3100,2845,1684 \mathrm{~cm}^{-1} ;{ }^{1} \mathrm{H} \mathrm{NMR} 10.08(\mathrm{~s}, 1 \mathrm{H}), 7.50(\mathrm{~d}, \mathrm{~J}=2.7$, $1 \mathrm{H}), 7.44(\mathrm{dd}, \mathrm{J}=4.8,3.0,1 \mathrm{H}), 7.41(\mathrm{~d}, \mathrm{~J}=8.5,1 \mathrm{H}), 7.24(\mathrm{dd}, \mathrm{J}=3.0,0.9,1 \mathrm{H}), 7.19(\mathrm{dd}, \mathrm{J}=8.5,2.7$, 1H), $7.17(\mathrm{dd}, \mathrm{J}=4.8,0.9,1 \mathrm{H}), 3.90(\mathrm{~s}, 3 \mathrm{H}) ;{ }^{13} \mathrm{C}$ NMR 192.2, 159.0, 137.9, 134.7, 133.5, 131.8, 129.4, 126.1, 124.5, 121.5, 109.7, 55.5; MS m/e $218\left(\mathrm{M}^{+}\right)$; HRMS m/e for $\mathrm{C}_{12} \mathrm{H}_{10} \mathrm{O}_{2} \mathrm{~S}$ calcd. $218.0402\left(\mathrm{M}^{+}\right)$, found 218.0399 .

3',4,4',5'-Tetramethoxy[1,1'-biphenyl]-2-carboxaldehyde (12e). Prepared according to the method of Fürstner ${ }^{17}$ employing 11a (0.7000 g, $\left.3.26 \mathrm{mmol}\right)$ and 3,4,5-trimethoxyphenylboronic acid (10d) (1.0877 g, $4.89 \mathrm{mmol})$ to give $12 \mathrm{e}(0.7472 \mathrm{~g}, 76 \%$ yield) following flash chromatographic purification (3:1 petroleum ether : $\mathrm{Et}_{2} \mathrm{O}$ ), as a colorless solid, mp $134-136{ }^{\circ} \mathrm{C}$; IR (KBr) 2931, $1687,1604 \mathrm{~cm}^{-1} ;{ }^{1} \mathrm{H}$ NMR $9.96(\mathrm{~s}, 1 \mathrm{H}), 7.47(\mathrm{~d}, \mathrm{~J}=2.8,1 \mathrm{H}), 7.37(\mathrm{~d}, \mathrm{~J}=8.5,1 \mathrm{H}), 7.17(\mathrm{dd}, \mathrm{J}=8.5,2.8,1 \mathrm{H}), 6.52(\mathrm{~s}, 2 \mathrm{H})$, 3.89 (s, 3H), 3.88 (s, 3H), 3.87 (s, 6H); ${ }^{13} \mathrm{C}$ NMR 192.2, 159.1, 152.9, 139.0, 137.7, 134.5, 133.1, 131.7, 121.2, 109.8, 107.5, 60.9, 56.2, 55.5; MS m/e $\left(\mathrm{M}^{+}\right)$302; HRMS m/e for $\mathrm{C}_{17} \mathrm{H}_{18} \mathrm{O}_{5}$ calcd. 302.1154, found 302.1139.

2',3,3',4,4'-Pentamethoxy[1,1'-Biphenyl]-2-carboxaldehyde (12f). Prepared according to the method of Fürstner ${ }^{17}$ employing 11c (0.7937 g, $\left.3.24 \mathrm{mmol}\right)$ and 2,3,4-trimethoxyphenylboronic acid (10a) $(1.0993 \mathrm{~g}, 5.18 \mathrm{mmol})$ to give $\mathbf{1 2 f}(0.8712 \mathrm{~g}, 81 \%$ yield $)$ following flash chromatographic purification (4:1 petroleum ether : EtOAc), mp 117-119 ${ }^{\circ} \mathrm{C} ; \mathrm{IR}(\mathrm{KBr}){ }_{\max } 2938,1699,1593 \mathrm{~cm}^{-1} ;{ }^{1} \mathrm{H}$ NMR $10.15(\mathrm{~s}, 1 \mathrm{H}), 7.12(\mathrm{~d}, \mathrm{~J}=8.4,1 \mathrm{H}), 7.01(\mathrm{~d}, \mathrm{~J}=8.4,1 \mathrm{H}), 6.87(\mathrm{~d}, \mathrm{~J}=8.5,1 \mathrm{H}), 6.71(\mathrm{~d}, \mathrm{~J}=8.5$, 1H), $3.96(\mathrm{~s}, 3 \mathrm{H}), 3.93(\mathrm{~s}, 3 \mathrm{H}), 3.890(\mathrm{~s}, 3 \mathrm{H}), 3.887$ (s, 3H), 3.58 (s, 3H); ${ }^{13} \mathrm{C}$ NMR 191.1, 153.2, 152.0, $150.5,149.9,141.7,131.7,129.0,126.6,125.7,124.5,115.9,106.9,61.7,60.7,60.3,55.7,55.7$; MS m/e $332\left(\mathrm{M}^{+}\right)$; HRMS for $\mathrm{C}_{18} \mathrm{H}_{20} \mathrm{O}_{6}\left(\mathrm{M}^{+}\right)$calcd. 332.1260, found 332.1275.

\section{3-(2',3',4,4'-Tetramethoxybiphenyl-2-yl)-2-propyn-1-ol (13a). General Procedure A. To a} solution of 12a $(0.3378 \mathrm{~g}, 1.12 \mathrm{mmol})$ in $\mathrm{CH}_{2} \mathrm{Cl}_{2}(10 \mathrm{~mL})$ was added $\mathrm{CBr}_{4}(0.556 \mathrm{~g}, 1.68 \mathrm{mmol})$ and $\mathrm{PPh}_{3}(1.172 \mathrm{~g}, 4.47 \mathrm{mmol})$. After stirring for $4 \mathrm{~h}$, petroleum ether $(10 \mathrm{~mL})$ and iodomethane $(0.4 \mathrm{~mL})$ were added, and the mixture allowed to stir for $8 \mathrm{~h}$ ). The volatiles were removed under reduced pressure, and the residue filtered through silica gel, using 1:1 petroleum ether : $\mathrm{Et}_{2} \mathrm{O}$ as solvent). The 
filtrate was concentrated under reduced pressure to give the crude dibromide, which was used without further purification. The dibromide was dissolved in THF $(20 \mathrm{~mL})$, and cooled to $-78{ }^{\circ} \mathrm{C}$. Butyllithium (1.08 $\mathrm{mL}$ of a $2.58 \mathrm{M}$ solution in hexanes, $2.79 \mathrm{mmol})$ was added, and stirring continued for $5 \mathrm{~h}$. A suspension of paraformaldehyde $(0.4 \mathrm{~g}$, excess $)$ in THF $(5 \mathrm{~mL})$ was added, and the reaction stirred for 8 $\mathrm{h}$ as the mixture gradually warmed to room temperature. A conventional extractive workup followed by flash chromatography (1:2 petroleum ether : $\left.\mathrm{Et}_{2} \mathrm{O}\right)$ gave 13a $(0.2919 \mathrm{~g}, 80 \%)$, as a viscous oil; IR $(\mathrm{KBr})$ ${ }_{\max } 3455 \mathrm{br}, 2937,2229 \mathrm{~cm}^{-1} ;{ }^{1} \mathrm{H}$ NMR $7.22(\mathrm{~d}, \mathrm{~J}=8.6,1 \mathrm{H}), 7.05(\mathrm{~d}, \mathrm{~J}=2.7,1 \mathrm{H}), 6.96(\mathrm{~d}, \mathrm{~J}=8.5$, $1 \mathrm{H}), 6.91(\mathrm{dd}, \mathrm{J}=8.6,2.7,1 \mathrm{H}), 6.68(\mathrm{dd}, \mathrm{J}=8.5,1 \mathrm{H}), 4.29(\mathrm{~s}, 2 \mathrm{H}), 3.90(\mathrm{~s}, 3 \mathrm{H}), 3.88(\mathrm{~s}, 3 \mathrm{H}), 3.82(\mathrm{~s}$, 3H), 3.61 (s, 3H), 2.30 (br, 1H) $;{ }^{13} \mathrm{C}$ NMR 158.2, 153.1, 151.6, 142.0, 133.3, 131.3, 127.1, 125.6, 123.2, 116.8, 114.8, 106.7, 89.6, 85.2, 61.0, 60.9, 55.9, 55.3, 51.4; MS m/e $328\left(\mathrm{M}^{+}\right)$; HRMS m/e for $\mathrm{C}_{19} \mathrm{H}_{20} \mathrm{O}_{5}$ calcd. $328.1311\left(\mathrm{M}^{+}\right)$, found 328.1311 .

3-(2',3',4'-Trimethoxybiphenyl-2-yl)-2-propyn-1-ol (13b). Reaction of aldehyde 12b (0.1514 g, $0.554 \mathrm{mmol})$ according to General Procedure A, afforded 13b (0.1293 g, 78\% yield) following preparative TLC (1:2 hexanes : $\left.\mathrm{Et}_{2} \mathrm{O}\right)$, as a viscous oil; IR $(\mathrm{KBr}){ }_{\max } 3379 \mathrm{br}, 2933,2227 \mathrm{~cm}^{-1} ;{ }^{1} \mathrm{H}$ NMR $7.52(\mathrm{dd}, \mathrm{J}=7.7,1.0,1 \mathrm{H}), 7.25-7.37(\mathrm{~m}, 3 \mathrm{H}), 6.98(\mathrm{~d}, \mathrm{~J}=8.5,1 \mathrm{H}), 6.71(\mathrm{~d}, \mathrm{~J}=8.6,1 \mathrm{H}), 4.29(\mathrm{~s}, 2 \mathrm{H})$, 3.91 (s, 3H), 3.91 (s, 3H), 3.63 (s, 3H), 2.07 (br, 1H); ${ }^{13} \mathrm{C}$ NMR 153.3, 151.5, 142.0, 140.8, 132.3, 130.2, 128.0, 127.5, 126.9, 125.3, 122.4, 106.8, 89.9, 85.2, 61.0, 60.9, 56.0, 51.4; MS m/e $298\left(\mathrm{M}^{+}\right)$; HRMS m/e for $\mathrm{C}_{18} \mathrm{H}_{18} \mathrm{O}_{4}$ calcd. 298.1205 $\left(\mathrm{M}^{+}\right)$, found 298.1201.

(3-Methoxy-3',5'-dimethylbiphenyl-2-yl)-2-propyn-1-ol (13c). Reaction of aldehyde 12c (0.8472 g, $3.53 \mathrm{mmol})$ according to General Procedure A, afforded 13c $(0.5389 \mathrm{~g}, 57 \%$ yield $)$ following flash chromatography $\left(2: 1\right.$ petroleum ether : $\left.\mathrm{Et}_{2} \mathrm{O}\right)$ as a viscous oil; IR $(\mathrm{KBr}){ }_{\max } 3400 \mathrm{br}, 2917,1603 \mathrm{~cm}^{-1} ;{ }^{1} \mathrm{H}$ NMR $7.31(\mathrm{~d}, \mathrm{~J}=8.5,1 \mathrm{H}), 7.20(\mathrm{~s}, 2 \mathrm{H}), 7.08(\mathrm{~d}, \mathrm{~J}=2.8,1 \mathrm{H}), 7.00(\mathrm{~s}, 1 \mathrm{H}), 6.96(\mathrm{dd}, \mathrm{J}=8.5,2.7,1 \mathrm{H})$, $4.37(\mathrm{~s}, 2 \mathrm{H}), 3.84(\mathrm{~s}, 3 \mathrm{H}), 2.38(\mathrm{~s}, 6 \mathrm{H}), 1.55(\mathrm{br}, 1 \mathrm{H}) ;{ }^{13} \mathrm{C}$ NMR 158.3, 140.0, 137.3, 136.9, 130.6, 128.7, 127.1, 121.6, 117.4, 115.4, 89.8, 85.6, 55.4, 51.6, 21.3; MS m/e $266\left(\mathrm{M}^{+}\right)$; HRMS m/e for $\mathrm{C}_{18} \mathrm{H}_{18} \mathrm{O}_{2}$ calcd. $266.1307\left(\mathrm{M}^{+}\right)$, found 266.1294. 
(14). Reaction of aldehyde 12d (0.0876 g, $0.401 \mathrm{mmol})$ according to General Procedure A gave, in order of elution, $14(0.0452 \mathrm{~g}, 53 \%$ yield $)$ and $\mathbf{1 3 d}(0.0389 \mathrm{~g}, 40 \%$ yield $)$, following preparative TLC (1:1 petroleum ether : $\left.\mathrm{Et}_{2} \mathrm{O}\right) ; \mathbf{1 4}$ as a colorless solid, mp $118-120{ }^{\circ} \mathrm{C}$; IR $(\mathrm{KBr}){ }_{\max } 2956,1621 \mathrm{~cm}^{-1} ;{ }^{1} \mathrm{H}$ NMR $8.26(d, J=8.8,1 H), 7.92(d, J=5.4,1 H), 7.87(d, J=8.7,1 H), 7.67(d, J=8.7,1 H), 7.58(d, J$ $=5.4,1 \mathrm{H}), 72.6-7.31(\mathrm{~m}, 2 \mathrm{H}), 3.97(\mathrm{~s}, 3 \mathrm{H}) ;{ }^{13} \mathrm{C} \mathrm{NMR} 157.2,136.1,135.4,132.2,125.9,125.1,124.5$, 124.3, 121.7, 121.2, 118.0, 107.6, 55.4; MS m/e $214\left(\mathrm{M}^{+}\right)$; HRMS for $\mathrm{C}_{13} \mathrm{H}_{10} \mathrm{O}_{2} \mathrm{~S}\left(\mathrm{M}^{+}\right)$calcd. 214.0452, found 214.0454. 13d as a viscous oil; IR (KBr) ${ }_{\text {max }} 3384 \mathrm{br}, 2928,2228 \mathrm{~cm}^{-1} ;{ }^{1} \mathrm{H}$ NMR $7.54(\mathrm{dd}, \mathrm{J}=$ 3.0, 1.4, 1H), $7.42(\mathrm{dd}, \mathrm{J}=5.0,1.4,1 \mathrm{H}), 7.34(\mathrm{~d}, \mathrm{~J}=8.6,1 \mathrm{H}), 7.34(\mathrm{dd}, \mathrm{J}=5.0,3.0,1 \mathrm{H}), 7.06(\mathrm{~d}, \mathrm{~J}=$ 2.7, $1 \mathrm{H}), 6.93(\mathrm{dd}, \mathrm{J}=8.6,2.7,1 \mathrm{H}) 4.46(\mathrm{~d}, \mathrm{~J}=4.2,2 \mathrm{H}), 3.84(\mathrm{~s}, 3 \mathrm{H}), 1.61(\mathrm{br}, 1 \mathrm{H}) ;{ }^{13} \mathrm{C}$ NMR 158.2, $140.5,131.2,130.2,128.5,124.7,122.6,121.2,117.7,115.6,90.2,85.7,55.4,51.8 ; \mathrm{MS} \mathrm{m} / \mathrm{e} 244\left(\mathrm{M}^{+}\right)$; HRMS m/e for $\mathrm{C}_{14} \mathrm{H}_{12} \mathrm{O}_{2} \mathrm{~S}$ calcd. $244.0558\left(\mathrm{M}^{+}\right)$, found 244.0550 .

3-(3',4,4',5'-Tetramethoxybiphenyl-2-yl)-2-propyn-1-ol (13e). Reaction of aldehyde 12e (0.4674 g, $1.55 \mathrm{mmol})$ according to General Procedure A, gave 13e (0.3115 g, 61\% yield), following flash chromatography (1:1 petroleum ether : $\left.\mathrm{Et}_{2} \mathrm{O}\right)$, as a viscous oil; IR (KBr) 3500 (br), 2935, 2224, $1602 \mathrm{~cm}^{-}$ ${ }^{1} ;{ }^{1} \mathrm{H}$ NMR $7.29(\mathrm{~d}, \mathrm{~J}=8.6,1 \mathrm{H}), 7.06(\mathrm{~d}, \mathrm{~J}=2.7,1 \mathrm{H}), 6.92(\mathrm{dd}, \mathrm{J}=8.6,2.7,1 \mathrm{H}), 6.79(\mathrm{~s}, 2 \mathrm{H}), 4.38(\mathrm{~s}$, 2H), $3.88(\mathrm{~s}, 3 \mathrm{H}), 3.87(\mathrm{~s}, 6 \mathrm{H}), 3.81(\mathrm{~s}, 3 \mathrm{H}), 2.33$ (br s, 1H); ${ }^{13} \mathrm{C}$ NMR 158.1, 152.5, 137.0, 136.1, $135.4,130.3,121.3,117.6,115.2,106.4,90.3,84.8,60.7,56.0,55.2,51.2 ; \mathrm{MS}$ m/e $\left(\mathrm{M}^{+}\right) 328 ; \mathrm{HRMS}$ $\mathrm{m} / \mathrm{e}$ for $\mathrm{C}_{19} \mathrm{H}_{20} \mathrm{O}_{5}$ calcd. 328.1311, found 328.1308.

3-(2'3,3',4,4'-Pentamethoxybiphenyl-2-yl)-2-propyn-1-ol (13f). Reaction of aldehyde $12 f(0.8629$ g, $2.60 \mathrm{mmol})$ according to General Procedure A, gave $13 f(0.5257 \mathrm{~g}, 56 \%$ yield $)$, flash chromatography (1:1 hexanes : $\left.\mathrm{Et}_{2} \mathrm{O}\right)$, as a viscous oil; IR $(\mathrm{KBr}) 3509$ (br), 2936, 2227, $1591 \mathrm{~cm}^{-1} ;{ }^{1} \mathrm{H}$ NMR $6.96(\mathrm{~d}, \mathrm{~J}=$ $8.5,1 \mathrm{H}), 6.91(\mathrm{~d}, \mathrm{~J}=8.6,1 \mathrm{H}), 6.88(\mathrm{~d}, \mathrm{~J}=8.5,1 \mathrm{H}), 6.64(\mathrm{~d}, \mathrm{~J}=8.6,1 \mathrm{H}), 4.28(\mathrm{br} \mathrm{d}, \mathrm{J}=4.8,2 \mathrm{H}), 3.89$ (s, 3H), $3.85(\mathrm{~s}, 3 \mathrm{H}), 3.84(\mathrm{~s}, 3 \mathrm{H}), 3.83(\mathrm{~s}, 3 \mathrm{H}), 3.61(\mathrm{~s}, 3 \mathrm{H}), 2.88(\mathrm{br}, 1 \mathrm{H}) ;{ }^{13} \mathrm{C}$ NMR 152.8, 151.24, $151.17,149.9,141.6,133.9,126.7,125.4,125.3,117.4,112.0,106.4,94.3,80.3,60.7,60.61,60.57$, 55.6, 51.0; MS m/e $358\left(\mathrm{M}^{+}\right)$; HRMS m/e for $\mathrm{C}_{20} \mathrm{H}_{22} \mathrm{O}_{6}$ calcd. 358.1416, found 358.1404. 
3-Biphenyl-2-yl-2-propyn-1-ol (13g). To a solution of 2'-iodobiphenyl (0.10 mL, $0.57 \mathrm{mmol})$, $\mathrm{Pd}\left(\mathrm{PPh}_{3}\right)_{4}(10 \mathrm{mg})$ and $\mathrm{CuI}(20 \mathrm{mg})$ in degassed diisopropylamine $(5 \mathrm{~mL})$ was added propargyl alcohol $(0.15 \mathrm{~mL}, 2.6 \mathrm{mmol})$. After $20 \mathrm{~h}$ of stirring, the volatiles were removed under reduced pressure, the residue was filtered through silica with $\mathrm{Et}_{2} \mathrm{O}$, and the volatiles again removed in vacuo. Preparative TLC in 2:1 hexanes:Et ${ }_{2} \mathrm{O}$ gave $0.1025 \mathrm{~g}$ of $\mathbf{1 3 g}$ (87\% yield), bp $135-140{ }^{\circ} \mathrm{C}$ (0.15 torr) (bulb-to-bulb); IR $(\mathrm{KBr})_{\max } 3054,3061,2925,2235 \mathrm{~cm}^{-1} ;{ }^{1} \mathrm{H}$ NMR $7.58(\mathrm{~d}, \mathrm{~J}=7.3,2 \mathrm{H}), 7.57(\mathrm{~d}, \mathrm{~J}=7.9,1 \mathrm{H}), 7.44$ (apparent t, $\left.\mathrm{J}_{\text {avs }}=7.5,2 \mathrm{H}\right), 7.35-7.41(\mathrm{~m}, 3 \mathrm{H}), 7,31(\mathrm{~m}, 1 \mathrm{H}), 4.35(\mathrm{~s}, 2 \mathrm{H}), 1.63$ (br s, 1H); ${ }^{13} \mathrm{C}$ NMR $(40$ $\left.{ }^{\circ} \mathrm{C}\right) 144.0,140.5,133.1,129.5,129.2,128.6,127.9,127.4,127.0,121.0,90.2,85.4,51.5 ; \mathrm{MS}$ m/e 208 $\left(\mathrm{M}^{+}\right) ; \mathrm{HRMS} \mathrm{m} / \mathrm{e}$ for $\mathrm{C}_{15} \mathrm{H}_{12} \mathrm{O}$ calcd. $208.0888\left(\mathrm{M}^{+}\right)$, found 208.0890.

Methyl 4-bromo-3-iodobenzoate. To a solution of 4-bromo-3-iodobenzoic acid (1.3227 g, 3.68 mmol) in methanol (40 mL) was added $\mathrm{H}_{2} \mathrm{SO}_{4}$ (10 drops). The mixture was heated to reflux for $12 \mathrm{~h}$. Following a conventional extractive workup $\left(\mathrm{Et}_{2} \mathrm{O}\right)$, and extraction of the $\mathrm{Et}_{2} \mathrm{O}$ layers with $\mathrm{NaOH}_{(\mathrm{aq})}$, the $\mathrm{Et}_{2} \mathrm{O}$ layers were dried over $\mathrm{MgSO}_{4}$, filtered and concentrated under reduced pressure. Flash chromatography (8:1 petroleum ether:Et $\left.{ }_{2} \mathrm{O}\right)$ afforded methyl 4-bromo-3-iodobenzoate $(1.2550 \mathrm{~g}, 91 \%$ yield) as a colorless solid, mp 70-71 ${ }^{\circ} \mathrm{C}$; IR (KBr) ${ }_{\text {max }} 3087,1726 \mathrm{~cm}^{-1} ;{ }^{1} \mathrm{H}$ NMR 8.46 (s, 1H), 7.81 (d, J $=8.2,1 \mathrm{H}), 7.66(\mathrm{~d}, \mathrm{~J}=8.2,1 \mathrm{H}), 3.90(\mathrm{~s}, 3 \mathrm{H}) ;{ }^{13} \mathrm{C} \mathrm{NMR} 164.8,141.1,135.2,132.5,130.12,130.08$, 101.0, 52.5; MS m/e (M+) 340/342; HRMS m/e for $\mathrm{C}_{8} \mathrm{H}_{6} \mathrm{BrIO}_{2}$ calcd. 339.8596, found 339.8603.

Methyl 4-bromo-3-(3-methoxyprop-1-ynyl)benzoate (15). To a solution of methyl 4-bromo-3iodobenzoate $(1.255 \mathrm{~g}, 3.21 \mathrm{mmol})$ and propargyl methyl ether $(0.47 \mathrm{~mL}, 5.9 \mathrm{mmol})$ in diisopropylamine $(5 \mathrm{~mL})$ was added $\mathrm{CuI}(0.0404 \mathrm{~g}, 0.212 \mathrm{mmol})$ and $\mathrm{Pd}\left(\mathrm{PPh}_{3}\right)_{4}(0.070 \mathrm{~g}, 0.061 \mathrm{mmol})$. After stir ring $12 \mathrm{~h}$, the mixture was subjected to a conventional extractive workup. Flash chromatography (5:1 petroleum ether:Et $\left.{ }_{2} \mathrm{O}\right)$ afforded $15(0.9599 \mathrm{~g}, 92 \%$ yield $)$ as yellow crystals, mp 42-43 ${ }^{\circ} \mathrm{C}$; IR (KBr) ${ }_{\max } 3033,2953,1739,1593 \mathrm{~cm}^{-1} ;{ }^{1} \mathrm{H}$ NMR $\quad{ }^{1} \mathrm{H}$ NMR $8.10(\mathrm{~d}, \mathrm{~J}=2.0,1 \mathrm{H}), 7.78$ $(\mathrm{dd}, \mathrm{J}=8.4,2.0,1 \mathrm{H}), 7.64(\mathrm{~d}, \mathrm{~J}=8.4,1 \mathrm{H}), 4.37(\mathrm{~s}, 2 \mathrm{H}), 3.89(\mathrm{~s}, 3 \mathrm{H}), 3.48(\mathrm{~s}, 3 \mathrm{H}) ;{ }^{13} \mathrm{C}$ NMR 165.6, 134.4, 132.5, 130.6, 130.1, 129.2, 125.2, 90.7, 84.0, 60.2, 57.7, 52.3; MS m/e 282/284 (M+); HRMS for $\mathrm{C}_{12} \mathrm{H}_{11} \mathrm{BrO}_{3}$ calcd. 283.9891, found 283.9900. 
Methyl 2',3',4'-trimethoxy-2-(3-methoxyprop-1-ynyl)biphenyl-4-carboxylate (16). A mixture of methyl 4-bromo-3-(3-methoxyprop-1-ynyl)benzoate $\quad(\mathbf{1 5}) \quad(0.0712 \quad \mathrm{~g}, \quad 0.251 \quad \mathrm{mmol}), \quad 2,3,4-$ trimethoxyphenylboronic acid (10a) $(0.1333 \mathrm{~g}, 0.629 \mathrm{mmol}), \mathrm{K}_{3} \mathrm{PO}_{4}(0.1590 \mathrm{~g}, 0.750 \mathrm{mmol}), \mathrm{Pd}_{2}(\mathrm{dba})_{3}$ (0.0046 g, $0.0050 \mathrm{mmol}), \mathrm{PCy}_{3}(0.0035 \mathrm{~g}, 0.012 \mathrm{mmol})$ in toluene $(10 \mathrm{~mL})$ was heated to $100{ }^{\circ} \mathrm{C}$ for 37 h. Following an extractive workup, radial chromatography $\left(5: 1\right.$ petroleum ether:Et $\left.{ }_{2} \mathrm{O}\right)$ afforded, in order of elution, recovered 15 (0.0218 g, 31\% recovery), and 16 (0.0493 g, 53\% yield) as a viscous oil; IR $(\mathrm{KBr})_{\max } 2936,1730,1605 \mathrm{~cm}^{-1} ;{ }^{1} \mathrm{H}$ NMR $8.22(\mathrm{~d}, \mathrm{~J}=1.8,1 \mathrm{H}), 8.00(\mathrm{dd}, \mathrm{J}=8.1,1.8,1 \mathrm{H}), 7.41(\mathrm{~d}, \mathrm{~J}=$ 8.1, 1H), $7.01(\mathrm{~d}, \mathrm{~J}=8.5,1 \mathrm{H}), 6.72(\mathrm{~d}, \mathrm{~J}=8.5,1 \mathrm{H}), 4.20(\mathrm{~s}, 2 \mathrm{H}), 3.94(\mathrm{~s}, 3 \mathrm{H}), 3.92(\mathrm{~s}, 3 \mathrm{H}), 3.91(\mathrm{~s}, 3 \mathrm{H})$, 3.66 (s, 3H), 3.23 (s, 3H); ${ }^{13} \mathrm{C}$ NMR 166.4, 153.8. 151.4, 145.3, 142.1, 133.7, 130.6, 128.9, 126.6, 125.2, $122.9,106.7,88.2,85.2,61.2,61.0,60.2,57.2,56.1,52.2 ; \mathrm{MS}$ m/e $370\left(\mathrm{M}^{+}\right)$; HRMS for $\mathrm{C}_{21} \mathrm{H}_{22} \mathrm{O}_{6}\left(\mathrm{M}^{+}\right)$ calcd. 370.1416 , found 370.1416 .

A solution of potassium tert-butoxide $(0.0351 \mathrm{~g}, 0.314 \mathrm{mmol})$ and (1,3-diisopropylimiazol-2ylidene)(3-chloropyridyl)palladium(II) dichloride $(0.0014 \mathrm{~g}, 1 \mathrm{~mol} \%)$ is isopropanol (1 $\mathrm{mL})$ was stirred for $10 \mathrm{~min}$. To this solution was added 2,3,4-trimethoxyphenylboronic acid (10a) $(0.0 .0975 \mathrm{~g}, 0.460$ $\mathrm{mmol})$ and 15 (0.0590 g, $0.209 \mathrm{mmol})$. After stirring for $12 \mathrm{~h}$, diethyl ether was added and reaction subjected to a conventional extractive workup $\left(\mathrm{Et}_{2} \mathrm{O}\right)$. Preparative TLC (5:1 petroleum ether: $\left.\mathrm{Et}_{2} \mathrm{O}\right)$ afforded 16 (0.0612 g, 79\% yield).

\section{Hexacarbonyl $\left[\mu\right.$ - ${ }^{4}$-(3-acetoxy-(2',3',4,4'-tetramethoxybiphenyl-2-yl)-1-propyne) $]$ dicobalt (8a).}

General Procedure B. To alcohol 13a $(0.2875 \mathrm{~g}, 0.876 \mathrm{mmol})$ at $0{ }^{\circ} \mathrm{C}$ was added acetic anhydride $(1$ $\mathrm{mL})$ and pyridine $(1 \mathrm{~mL})$. The solution was stirred $4 \mathrm{~h}$, as the solution came to room temperature. The volatiles were removed under reduced pressure, and the residue dissolved in $\mathrm{CH}_{2} \mathrm{Cl}_{2}(25 \mathrm{~mL})$. An unweighed amount of octacarbonyldicobalt (excess) was added and the mixture stirred 4h. After concentration under reduced pressure, flash chromatography $(100 \%$ petroleum ether $-5: 1$ petroleum ether : $\left.\mathrm{Et}_{2} \mathrm{O}\right)$ gave acetate complex $8 \mathbf{a}\left(0.4952 \mathrm{~g}, 86 \%\right.$ yield) as a red-brown solid, mp $122-124{ }^{\circ} \mathrm{C}$; IR $(\mathrm{KBr})_{\max } 2919,2090,2050,2018,1743 \mathrm{~cm}^{-1} ;{ }^{1} \mathrm{H}$ NMR $7.30(\mathrm{~d}, \mathrm{~J}=2.5,1 \mathrm{H}), 7.00(\mathrm{~d}, \mathrm{~J}=8.5,1 \mathrm{H})$, $6.88(\mathrm{dd}, \mathrm{J}=8.5,2.5,1 \mathrm{H}), 6.80(\mathrm{~d}, \mathrm{~J}=8.5,1 \mathrm{H}), 6.73(\mathrm{~d}, \mathrm{~J}=8.5,1 \mathrm{H}), 4.58(\mathrm{~d}, \mathrm{~J}=15.0,1 \mathrm{H}), 3.97(\mathrm{~d}, \mathrm{~J}=$ 
15.0, 1H), $3.96(\mathrm{~s}, 3 \mathrm{H}), 3.93(\mathrm{~s}, 3 \mathrm{H}), 3.86(\mathrm{~s}, 3 \mathrm{H}), 3.59(\mathrm{~s}, 3 \mathrm{H}), 2.02(\mathrm{~s}, 3 \mathrm{H}) ;{ }^{13} \mathrm{C}$ NMR 199.4, 199.3, $170.3,159.2,153.9,151.2,142.4,137.6,131.8,129.4,128.2,125.4,118.0,114.5,107.3,92.5,88.7$, 64.4, 60.7, 60.4, 56.2, 55.0, 20.2; MS m/e 600 (M+2CO), $572\left(\mathrm{M}^{+}-3 \mathrm{CO}\right), 488\left(\mathrm{M}^{+}-6 \mathrm{CO}\right)$; Anal. Calcd. for $\mathrm{C}_{27} \mathrm{H}_{22} \mathrm{Co}_{2} \mathrm{O}_{12} \mathrm{C}, 49.41 ; \mathrm{H}, 3.38$. Found $\mathrm{C}, 49.68 ; \mathrm{H}, 3.38$.

\section{Hexacarbonyl[ $\mu$ - ${ }^{4}$-(3-acetoxy-(2',3',4'-trimethoxybiphenyl-2-yl)-1-propyne $]$ dicobalt}

$(8 b)$.

Subjecting 13b $(0.2140 \mathrm{~g}, 0.407 \mathrm{mmol})$ to General Procedure B afforded 18b $(0.2140 \mathrm{~g}, 84 \%$ yield $)$ following flash chromatographic purification $\left(10: 1-4: 1\right.$ petroleum ether:Et $\left.{ }_{2} \mathrm{O}\right)$, as a red-brown solid, mp 108-110 ${ }^{\circ} \mathrm{C}$; IR (KBr) ${ }_{\max } 2938,2090,2052,2022,1745 \mathrm{~cm}^{-1} ;{ }^{1} \mathrm{H}$ NMR $7.76(\mathrm{~d}, \mathrm{~J}=7.8,1 \mathrm{H}), 7.40$ (apparent t, J = 7.4, 1H), 7.31 (apparent $\mathrm{t}, \mathrm{J}=7.3,1 \mathrm{H}), 7.11(\mathrm{~d}, \mathrm{~J}=7.5,1 \mathrm{H}), 6.82(\mathrm{~d}, \mathrm{~J}=8.4,1 \mathrm{H}), 6.75$ $(\mathrm{d}, \mathrm{J}=8.4,1 \mathrm{H}), 4.59(\mathrm{~d}, \mathrm{~J}=14.5,1 \mathrm{H}), 3.98(\mathrm{~d}, \mathrm{~J}=14.5,1 \mathrm{H}), 3.98(\mathrm{~s}, 3 \mathrm{H}), 3.94(\mathrm{~s}, 3 \mathrm{H}), 3.60(\mathrm{~s}, 3 \mathrm{H})$, 2.03 (s, 3H); ${ }^{13} \mathrm{C}$ NMR 199.3, 170.4, 154.1, 151.5, 142.6, 137.1, 136.6, 134.3, 130.9, 128.6, 128.1, 127.4, 125.0, 107.5, 92.6, 88.6, 64.6, 60.8, 60.5, 56.4. 20.2 ; MS m/e $570\left(\mathrm{M}^{+}-2 \mathrm{CO}\right), 542\left(\mathrm{M}^{+}-3 \mathrm{CO}\right)$, $458\left(\mathrm{M}^{+}-6 \mathrm{CO}\right)$; HRMS for $\mathrm{C}_{26} \mathrm{H}_{20} \mathrm{Co}_{2} \mathrm{O}_{11}$ calcd. 597.9720 ( $\left.\mathrm{M}^{+}-\mathrm{CO}\right)$, found 597.9741.

\section{Hexacarbonyl[ $\mu$ - ' -(3-acetoxy-(3-methoxy-3',5'-dimethylbiphenyl-2-yl)-1-propyne]dicobalt (8c).}

Subjecting 13c $(0.0608 \mathrm{~g}, 0.228 \mathrm{mmol})$ to General Procedure B afforded acetate complex 8c $(0.1232 \mathrm{~g}$, $91 \%$ yield) of acetate complex (91\% yield) following flash chromatography (10:1 petroleum ether : $\mathrm{Et}_{2} \mathrm{O}$ ), as a red-brown solid, $\mathrm{mp} 250{ }^{\circ} \mathrm{C}$ (dec.); IR (KBr) ${ }_{\max } 2961,2090,2058,1998,1748 \mathrm{~cm}^{-1} ;{ }^{1} \mathrm{H}$ NMR $7.28(\mathrm{~d}, \mathrm{~J}=2.7,1 \mathrm{H}), 7.08(\mathrm{~s}, 1 \mathrm{H}), 7.04(\mathrm{~d}, \mathrm{~J}=8.4,1 \mathrm{H}), 6.89(\mathrm{dd}, \mathrm{J}=8.4,2.7,1 \mathrm{H}), 6.86(\mathrm{~s}, 2 \mathrm{H})$, 4.02 (s, 2H), 3.87 (s, 3H), 2.37 (s, 6H), 2.04 (s, 3H); ${ }^{13} \mathrm{C}$ NMR 199.4, 170.4, 159.2, 141.6, 138.2, 136.4, $134.2,131.1,129.2,127.4,118.0,114.6,93.3,88.9,64.2,55.2,21.1,20.3 ; \mathrm{MS}$ m/e $566\left(\mathrm{M}^{+}-\mathrm{CO}\right), 510$ ( $\left.\mathrm{M}^{+}-3 \mathrm{CO}\right), 426\left(\mathrm{M}^{+}-6 \mathrm{CO}\right)$; HRMS m/e for $\mathrm{C}_{26} \mathrm{H}_{20} \mathrm{Co}_{2} \mathrm{O}_{9}$ calcd. $565.9822\left(\mathrm{M}^{+}-\mathrm{CO}\right)$, found 565.9811.

\section{Hexacarbonyl $\left[\mu\right.$ - ${ }^{4}$-(3-Acetoxy-1-(5-methoxy-2-(3-thienyl)phenyl)-1-propyne $]$ dicobalt}

(8d).

Subjecting 13d $(0.0418 \mathrm{~g}, 0.171 \mathrm{mmol})$ to General Procedure B gave acetate complex 8d $(0.0756,77 \%$ yield) following flash chromatography (10:1 petroleum ether : $\mathrm{Et}_{2} \mathrm{O}$ ), as a red-brown solid, mp 104-105 ${ }^{\circ} \mathrm{C}$; IR (KBr) ${ }_{\text {max }} 2941,2090,2055,2021,1745 \mathrm{~cm}^{-1} ;{ }^{1} \mathrm{H}$ NMR $7.46(\mathrm{dd}, \mathrm{J}=4.7,3.1,1 \mathrm{H}), 7.28(\mathrm{~d}, \mathrm{~J}=$ 2.6, 1H), $7.15(\mathrm{~m}, 1 \mathrm{H}), 7.06(\mathrm{~d}, \mathrm{~J}=8.4,1 \mathrm{H}), 7.02(\mathrm{~d}, \mathrm{~J}=4.7,1 \mathrm{H}), 6.88(\mathrm{dd}, \mathrm{J}=8.4,2.6,1 \mathrm{H}), 4.30(\mathrm{~s}$, 
2H), 3.87 (s, 3H), 2.05 (s, 3H); ${ }^{13} \mathrm{C}$ NMR 199.3, 170.5, 159.6, 141.9, 137.2, 131.8, 130.1, 128.3, 126.1, 123.8, 118.3, 114.7, 93.1, 88.4, 63.9, 55.2, 20.3; MS m/e $544\left(\mathrm{M}^{+}-\mathrm{CO}\right), 488\left(\mathrm{M}^{+}-3 \mathrm{CO}\right), 404\left(\mathrm{M}^{+}-6 \mathrm{CO}\right)$; HRMS m/e for $\mathrm{C}_{22} \mathrm{H}_{14} \mathrm{Co}_{2} \mathrm{O}_{9} \mathrm{~S}$ calcd. $543.9073\left(\mathrm{M}^{+}-\mathrm{CO}\right)$, found 543.9078.

Hexacarbonyl $\left[\mu\right.$ - ${ }^{4}$-(3-acetoxy-(,3',4,4',5'-tetramethoxybiphenyl-2-yl)-1-propyne)]dicobalt (8e). Subjecting 13e $(0.2809 \mathrm{~g}, 0.855 \mathrm{mmol})$ to General Procedure B afforded 8e $(0.4722 \mathrm{~g}, 84 \%$ yield $)$ following flash chromatographic purification $\left(3: 1\right.$ petroleum ether : $\left.\mathrm{Et}_{2} \mathrm{O}\right)$, as a red-brown solid; mp 126$128{ }^{\circ} \mathrm{C}$; IR (KBr) ${ }_{\max } 2938,2090,2010,1974,1748 \mathrm{~cm}^{-1} ; 1 \mathrm{H}$ NMR $7.27(\mathrm{~d}, \mathrm{~J}=2.6,1 \mathrm{H}), 7.05(\mathrm{~d}, \mathrm{~J}=$ 8.4, 1H), $6.89(\mathrm{dd}, \mathrm{J}=8.4,2.6,1 \mathrm{H}), 6.42(\mathrm{~s}, 2 \mathrm{H}), 4.22(\mathrm{~s}, 2 \mathrm{H}), 3.93(\mathrm{~s}, 3 \mathrm{H}), 3.86(\mathrm{~s}, 3 \mathrm{H}), 3.82(\mathrm{~s}, 6 \mathrm{H})$, 2.02 (s, 3H); ${ }^{13} \mathrm{C}$ NMR 199.3, 170.3, 159.4, 153.2, 137.7, 137.3, 136.4, 133.6, 131.2, 117.7, 114.7, 106.9, 93.1, 88.3, 63.9, 61.1, 56.1, 55.1, 20.2; MS m/e $628\left(\mathrm{M}^{+}-\mathrm{CO}\right), 600\left(\mathrm{M}^{+}-2 \mathrm{CO}\right), 572\left(\mathrm{M}^{+}-3 \mathrm{CO}\right), 544$ $\left(\mathrm{M}^{+}-4 \mathrm{CO}\right), 516\left(\mathrm{M}^{+}-5 \mathrm{CO}\right), 488\left(\mathrm{M}^{+}-6 \mathrm{CO}\right)$; HRMS for $\mathrm{C}_{27} \mathrm{H}_{22} \mathrm{Co}_{2} \mathrm{O}_{12}$ calcd $\left(\mathrm{M}^{+}-\mathrm{CO}\right) 627.9826$, found 627.9802.

Hexacarbonyl[ $\mu$ - ${ }^{4}$-(3-acetoxy-(2',3,3',4,4'-pentamethoxybiphenyl-2-yl)-1-propyne)]dicobalt (8f). Subjecting 13f (0.5231 g, $1.46 \mathrm{mmol})$ to General Procedure B afforded 8f $(0.9818 \mathrm{~g}$, $98 \%$ yield $)$ following flash chromatographic purification (4:1 petroleum ether : $\left.\mathrm{Et}_{2} \mathrm{O}\right)$, as a red-brown solid, mp 120$121{ }^{\circ} \mathrm{C}$; IR (KBr) ${ }_{\text {max }} 3002,2939,2088,2009,1743 \mathrm{~cm}^{-1} ;{ }^{1} \mathrm{H}$ NMR $6.95(\mathrm{~d}, \mathrm{~J}=8.4,1 \mathrm{H}), 6.80(\mathrm{~d}, \mathrm{~J}=$ 8.4, 1H), $6.73(\mathrm{~d}, \mathrm{~J}=8.4,1 \mathrm{H}), 6.71(\mathrm{~d}, \mathrm{~J}=8.4,1 \mathrm{H}), 4.53(\mathrm{~d}, \mathrm{~J}=14.5,1 \mathrm{H}), 4.07(\mathrm{~s}, 3 \mathrm{H}), 3.95(\mathrm{~s}, 3 \mathrm{H})$, $3.92(\mathrm{~s}, 3 \mathrm{H}), 3.90(\mathrm{~s}, 3 \mathrm{H}), 3.83(\mathrm{~d}, \mathrm{~J}=14.5,1 \mathrm{H}), 3.61(\mathrm{~s}, 3 \mathrm{H}), 2.00(\mathrm{~s}, 3 \mathrm{H}) ;{ }^{13} \mathrm{C} \mathrm{NMR} 199.8,170.4$, $153.9,151.6,151.4,149.5,142.4,131.3,128.84,128.79,125.6,125.4,112.2,107.2,94.3,81.0,65.4$, 60.6, 60.3, 59.9, 56.2, 55.7, 20.2; MS m/e $630\left(\mathrm{M}^{+}-2 \mathrm{CO}\right), 602\left(\mathrm{M}^{+}-3 \mathrm{CO}\right), 518\left(\mathrm{M}^{+}-6 \mathrm{CO}\right), 400\left(\mathrm{M}^{+}-\right.$ $\left.\mathrm{Co}_{2}(\mathrm{CO})_{6}\right)$; HRMS for $\mathrm{C}_{28} \mathrm{H}_{24} \mathrm{Co}_{2} \mathrm{O}_{13}\left(\mathrm{M}^{+}-2 \mathrm{CO}\right)$ calcd. 629.9997, found 629.9995.

Hexacarbonyl[ $\mu$ - ${ }^{4}$-(3-Acetoxy-1-biphenyl-2-yl)-1-propyne]dicobalt (8g). Subjecting 13g (0.0799 g, $0.384 \mathrm{mmol})$ to General Procedure B gave acetate complex $\mathbf{8 g}(0.1763 \mathrm{~g}, 86 \%$ yield $)$ following flash chromatography (50:1 petroleum ether : $\left.\mathrm{Et}_{2} \mathrm{O}\right)$, as a red-brown oil which gradually solidified, mp 99-101 ${ }^{\circ} \mathrm{C}$; IR (KBr) ${ }_{\max } 3073,2977,2087,2055,2006,1749 \mathrm{~cm}^{-1} ;{ }^{1} \mathrm{H}$ NMR $7.77(\mathrm{~d}, \mathrm{~J}=7.8,1 \mathrm{H}), 7.45-7.52$ $(\mathrm{m}, 3 \mathrm{H}), 7.42$ (apparent $\left.\mathrm{t}, \mathrm{J}_{\text {ave }}=7.6,1 \mathrm{H}\right), 7.34\left(\right.$ apparent $\left.\mathrm{t}, \mathrm{J}_{\text {ave }}=7.3,1 \mathrm{H}\right), 7.26-7.30(\mathrm{~m}, 2 \mathrm{H}), 7.14(\mathrm{~d}, \mathrm{~J}=$ 
7.8, 1H), 4.02 (s, 2H), 2.02 (s, 3H); ${ }^{13} \mathrm{C}$ NMR 199.3, 170.4, 142.2, 141.2, 135.3, 134.3, 130.2, 129.3, 128.6, 128.3, 128.0, 127.6, 93.1, 88.4, 64.1, 20.3; MS m/e $480\left(\mathrm{M}^{+}-2 \mathrm{CO}\right), 452\left(\mathrm{M}^{+}-3 \mathrm{CO}\right), 396\left(\mathrm{M}^{+}-\right.$ 5CO), $368\left(\mathrm{M}^{+}-6 \mathrm{CO}\right)$; Anal. Calcd. for $\mathrm{C}_{23} \mathrm{H}_{14} \mathrm{Co}_{2} \mathrm{O}_{8}$. C, 51.52; H, 2.63. Found C, 51.75; H, 2.51.

\section{Hexacarbonyl $\left[\mu\right.$ - ${ }^{4}$-(3-methoxy-(4-carbomethoxy-2',3',4'-trimethoxybiphenyl-2-yl)-1-}

propyne)]dicobalt $(\mathbf{8 h})$. To a solution of $16(0.1223 \mathrm{~g}, 0.330 \mathrm{mmol})$ in $\mathrm{CH}_{2} \mathrm{Cl}_{2}$ at $0{ }^{\circ} \mathrm{C}$ was added octacarbonyldicobalt (excess). The cooling bath was removed and the mixture allowed to stir for $2 \mathrm{~h}$. After concentration under reduced pressure, flash chromatography $\left(3: 1\right.$ petroleum ether : $\left.\mathrm{Et}_{2} \mathrm{O}\right)$ afforded 8h $\left(0.1797\right.$ g, 83\% yield) as a red-brown solid, mp 143-144 ${ }^{\circ} \mathrm{C}$; IR (KBr) ${ }_{\max } 2956,2091,2039,2009$, $1726 \mathrm{~cm}^{-1} ;{ }^{1} \mathrm{H}$ NMR $8.45(\mathrm{~d}, \mathrm{~J}=1.7,1 \mathrm{H}), 7.93(\mathrm{dd}, \mathrm{J}=7.9,1.7,1 \mathrm{H}), 7.18(\mathrm{~d}, \mathrm{~J}=7.9,1 \mathrm{H}), 6.80(1 / 2$ AB quartet, $\mathrm{J}=8.5,1 \mathrm{H}), 6.74(1 / 2 \mathrm{AB}$ quartet, $\mathrm{J}=8.5,1 \mathrm{H}), 3.97(\mathrm{~s}, 3 \mathrm{H}), 3.96(\mathrm{~s}, 3 \mathrm{H}), 3.94(\mathrm{~s}, 3 \mathrm{H}), 3.87$ $(\mathrm{d}, \mathrm{J}=13.4,1 \mathrm{H}), 3.59(\mathrm{~s}, 3 \mathrm{H}), 3.30(\mathrm{~s}, 3 \mathrm{H}), 3.25(\mathrm{~d}, \mathrm{~J}=13.4,1 \mathrm{H}) ;{ }^{13} \mathrm{C}$ NMR 199.4, 166.6, 154.0, 151.3, $142.4,141.3,137.8,135.7,131.2,130.1,128.1,127.8,124.9,107.1,95.7,86.0,71.7,60.8,60.6,58.6$, 56.3, 52.3; MS m/e $572\left(\mathrm{M}^{+}-3 \mathrm{CO}\right), 516\left(\mathrm{M}^{+}-5 \mathrm{CO}\right)$; HRMS for $\mathrm{C}_{27} \mathrm{H}_{22} \mathrm{Co}_{2} \mathrm{O}_{12}$ calcd $\left(\mathrm{M}^{+}-3 \mathrm{CO}\right)$ 571.9928, found 571.9924.

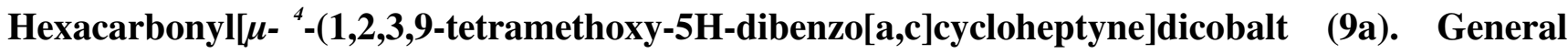

Procedure C. To a solution of $8 \mathbf{a}(0.1193 \mathrm{~g}, 0.182 \mathrm{mmol})$ in $\mathrm{CH}_{2} \mathrm{Cl}_{2}(35 \mathrm{~mL})$ at $0{ }^{\circ} \mathrm{C}$ was added diisopropylethylamine (48 $\mu \mathrm{L}, 1.5$ equiv) and $\mathrm{BF}_{3}-\mathrm{OEt}_{2}(69 \mu \mathrm{L}, 3.0$ equiv). The cooling bath was removed and the reaction allowed to stir for $6 \mathrm{~h}$, at which time consumption of starting material was complete. Following an extractive workup, flash chromatography (5:1 petroleum ether : $\left.\mathrm{Et}_{2} \mathrm{O}\right)$ afforded 9a $\left(0.0766 \mathrm{~g}, 71 \%\right.$ yield), as a red-brown oil which gradually solidified, mp $117-119{ }^{\circ} \mathrm{C}$; IR $(\mathrm{KBr}){ }_{\max }$ $\mathrm{cm}^{-1} ;{ }^{1} \mathrm{H}$ NMR $7.57(\mathrm{~d}, \mathrm{~J}=8.5,1 \mathrm{H}), 7.21(\mathrm{~d}, \mathrm{~J}=3.0,1 \mathrm{H}), 6.92(\mathrm{dd}, \mathrm{J}=8.5,2.5,1 \mathrm{H}), 6.65(\mathrm{~s}, 1 \mathrm{H})$, $4.01(\mathrm{~d}, \mathrm{~J}=14.0,1 \mathrm{H}), 3.91(\mathrm{~s}, 3 \mathrm{H}), 3.90(\mathrm{~s}, 3 \mathrm{H}), 3.82(\mathrm{~s}, 3 \mathrm{H}), 3.63(\mathrm{~d}, \mathrm{~J}=14.0,1 \mathrm{H}) 3.38(\mathrm{~s}, 3 \mathrm{H}) ;{ }^{13} \mathrm{C}$ NMR 199.3, 198.7, 158.9, 152.9, 152.2, 142.3, 138.9, 137.2, 133.7, 126.1, 124.8, 116.5, 112.0, 107.7, 105.4, 93.0, 60.9, 60.2, 56.0, 55.1, 39.8; MS m/e $596\left(\mathrm{M}^{+}\right), 540\left(\mathrm{M}^{+}-2 \mathrm{CO}\right), 512\left(\mathrm{M}^{+}-3 \mathrm{CO}\right), 484\left(\mathrm{M}^{+}-\right.$ 4CO); HRMS m/e for $\mathrm{C}_{25} \mathrm{H}_{18} \mathrm{Co}_{2} \mathrm{O}_{10}$ calcd. $539.9666\left(\mathrm{M}^{+}-2 \mathrm{CO}\right)$, found 539.9642 . 


\section{Hexacarbonyl[ $\mu$ - ${ }^{4}-(1,2,3-T$ rimethoxy-5H-dibenzo[a,c]cycloheptyne]dicobalt $\quad$ (9b). Subjecting}

acetate complex $\mathbf{8 b}(0.0848 \mathrm{~g}, 0.135 \mathrm{mmol})$ to General Procedure C $(16 \mathrm{~h})$, followed by flash chromatography (5:1 pet ether:Et $\left.{ }_{2} \mathrm{O}\right)$ afforded $9 \mathrm{~b}(0.0452 \mathrm{~g}, 59 \%$ yield, $66 \%$ yield based on recovered starting material) followed by recovered $\mathbf{8 b}(0.0085 \mathrm{~g}, 10 \%$ recovery). 9b, red-brown solid, mp 116-118 ${ }^{\circ} \mathrm{C}$; IR (KBr) ${ }_{\max } 2057,2934,2090,2051 \mathrm{~cm}^{-1} ;{ }^{1} \mathrm{H}$ NMR $7.69(\mathrm{dd}, \mathrm{J}=7.4,1.3,1 \mathrm{H}), 7.63(\mathrm{dd}, \mathrm{J}=7.6$, 1.3, 1H), 7.38 (apparent dt, $\mathrm{J}=1.3,7.4,1 \mathrm{H}), 7.35$ (apparent dt, $\mathrm{J}=1.3,7.6,1 \mathrm{H}), 6.67(\mathrm{~s}, 1 \mathrm{H}), 4.03(\mathrm{~d}, \mathrm{~J}$ $=14.0,1 \mathrm{H}), 3.94(\mathrm{~s}, 3 \mathrm{H}), 3.84(\mathrm{~s}, 3 \mathrm{H}), 3.66(\mathrm{~d}, \mathrm{~J}=14.0,1 \mathrm{H}), 3.39(\mathrm{~s}, 3 \mathrm{H}) ;{ }^{13} \mathrm{C}$ NMR 198.9 (br), 153.1 , $152.7,142.5,137.7,137.5,132.6,132.4,132.0,127.8,126.4,126.2,107.9,105.7,92.9,61.0,60.4,56.1$, 40.0; MS m/e $538\left(\mathrm{M}^{+}-1 \mathrm{CO}\right), 510\left(\mathrm{M}^{+}-2 \mathrm{CO}\right), 482\left(\mathrm{M}^{+}-3 \mathrm{CO}\right), 454\left(\mathrm{M}^{+}-4 \mathrm{CO}\right), 426\left(\mathrm{M}^{+}-5 \mathrm{CO}\right), 398\left(\mathrm{M}^{+}-\right.$ 6CO) ; HRMS m/e for $\mathrm{C}_{24} \mathrm{H}_{16} \mathrm{Co}_{2} \mathrm{O}_{9}$ calcd. 537.9509 ( $\left.\mathrm{M}^{+}-\mathrm{CO}\right)$, found 537.9492 .

\section{Hexacarbonyl[ $\mu$ - ${ }^{4}$-(9-methoxy-2,4-dimethyl-5H-dibenzo[a,c]cycloheptyne]dicobalt (9c).}

Subjecting acetate complex 8c $(0.1232 \mathrm{~g}, 0.207 \mathrm{mmol})$ to General Procedure C $(6 \mathrm{~h})$ gave 9c $(0.0938$ g, 85\% yield), following flash chromatography (50:1 petroleum ether : $\mathrm{Et}_{2} \mathrm{O}$ ), as a brown solid, mp 142$144{ }^{\circ} \mathrm{C}$; IR (KBr) ${ }_{\max } 2924,2090,2055,2025 \mathrm{~cm}^{-1} ;{ }^{1} \mathrm{H}$ NMR $\left(-30{ }^{\circ} \mathrm{C}\right) \quad 7.54(\mathrm{~d}, \mathrm{~J}=8.5,1 \mathrm{H}), 7.21(\mathrm{~d}, \mathrm{~J}=$ 3.0, 1H), $6.97-7.01(\mathrm{~m}, 3 \mathrm{H}), 4.49(\mathrm{~d}, \mathrm{~J}=14.0,1 \mathrm{H}), 3.93(\mathrm{~s}, 3 \mathrm{H}), 3.34(\mathrm{~d}, \mathrm{~J}=14.0,1 \mathrm{H}), 2.50(\mathrm{~s}, 3 \mathrm{H})$, 2.29 (s, 3H) ; ${ }^{13} \mathrm{C}$ NMR 199.1, 159.4, 140.6, 139.0, 136.20, 136.15, 134.1, 132.2, 132.0, 131.7, 130.0, 116.7, 113.5, 105.2, 92.7, 55.4, 32.9, 21.0, 20.9; MS m/e $534\left(\mathrm{M}^{+}\right), 450\left(\mathrm{M}^{+}-3 \mathrm{CO}\right), 394\left(\mathrm{M}^{+}-5 \mathrm{CO}\right)$; HRMS m/e for $\mathrm{C}_{24} \mathrm{H}_{16} \mathrm{Co}_{2} \mathrm{O}_{7}$ calcd. $533.9560\left(\mathrm{M}^{+}\right)$, found 533.9569.

Hexacarbonyl[$\left[\mu\right.$ - ${ }^{4}-(8-m e t h o x y-4 H-b e n z o[3,4]$ cycloheptyne[1,2-b]thiophene $]$ dicobalt (9d) and Hexacarbonyl $\left[\mu\right.$ - ${ }^{4}$-(8-methoxy-4H-benzo[3,4]cycloheptyne[1,2-c]thiophene $]$ dicobalt

Subjecting 8d (0.0654 g, $0.114 \mathrm{mmol})$ to General Procedure C (5 h) afforded 9d/9d' (0.0494 g, 82\% yield) following flash chromatography, as an inseparable 45:55 mixture, as a red-brown viscous oil; IR $(\mathrm{KBr})_{\max } 2937,2091,2067,2045,1603 \mathrm{~cm}^{-1} ;{ }^{1} \mathrm{H}$ NMR for 9d $7.53(\mathrm{~d}, \mathrm{~J}=8.5,1 \mathrm{H}), 7.24(\mathrm{~d}, \mathrm{~J}=2.8$, $1 \mathrm{H}), 7.18(\mathrm{~d}, \mathrm{~J}=5.3,1 \mathrm{H}), 7.13(\mathrm{~d}, \mathrm{~J}=5.3,1 \mathrm{H}), 6.95(\mathrm{dd}, \mathrm{J}=8.3,2.8,1 \mathrm{H}), 4.25(\mathrm{~s}, 2 \mathrm{H}), 3.900(\mathrm{~s}, 3 \mathrm{H})$; for 9d' $7.55(\mathrm{~d}, \mathrm{~J}=8.5,1 \mathrm{H}), 7.28(\mathrm{~d}, \mathrm{~J}=3.1,1 \mathrm{H}), 7.19(\mathrm{~d}, \mathrm{~J}=2.8,1 \mathrm{H}), 7.14$ (obscured d, 1H), 6.93 $(\mathrm{dd}, \mathrm{J}=8.3,2.81 \mathrm{H}), 4.17(\mathrm{~s}, 2 \mathrm{H}), 3.897(\mathrm{~s}, 3 \mathrm{H}) ;{ }^{13} \mathrm{C}$ NMR 199.0, 159.6, 159.1, 141.3, 139.5, 138.0, 
$137.2,136.9,135.7,131.1,130.2,129.9,127.1,127.0,125.4,121.9,121.5,117.3,117.1,114.0,113.8$, 100.9, 100.4, 92.5, 55.4, 34.8, 33.3; MS m/e $512\left(\mathrm{M}^{+}\right), 455\left(\mathrm{M}^{+}-2 \mathrm{CO}\right), 399\left(\mathrm{M}^{+}-4 \mathrm{CO}\right)$; HRMS m/e for $\mathrm{C}_{20} \mathrm{H}_{10} \mathrm{Co}_{2} \mathrm{O}_{7} \mathrm{~S}$ calcd. $511.8811\left(\mathrm{M}^{+}\right)$, found 511.8808 .

Hexacarbonyl $\left[\mu\right.$ - ${ }^{4}$-(2,3,4,9-tetramethoxy-5H-dibenzo[a,c]cycloheptyne]dicobalt (9e). Subjecting (8e) $(0.6621 \mathrm{~g}, 1.01 \mathrm{mmol})$ to General Procedure C (4.5 h) afforded 9e $(0.5457 \mathrm{~g}, 91 \%$ yield $)$ following flash chromatography $\left(7: 1\right.$ petroleum ether: $\left.\mathrm{Et}_{2} \mathrm{O}\right)$, as a red-brown solid; mp $138-140{ }^{\circ} \mathrm{C}$; IR $(\mathrm{KBr}){ }_{\max }$ 2938, 2091, 2051, 2023, $1601 \mathrm{~cm}^{-1} ;{ }^{1} \mathrm{H}$ NMR $7.47(\mathrm{~d}, \mathrm{~J}=8.7,1 \mathrm{H}), 7.22(\mathrm{~d}, \mathrm{~J}=2.7,1 \mathrm{H}), 6.98(\mathrm{dd}, \mathrm{J}=$ 8.7, 2.7, 1H), $6.67(\mathrm{~s}, 1 \mathrm{H}), 4.03\left(\mathrm{v}\right.$ br, 2H), $3.95(\mathrm{~s}, 3 \mathrm{H}), 3.91(\mathrm{~s}, 6 \mathrm{H}), 3.84(\mathrm{~s}, 3 \mathrm{H})$; ${ }^{13} \mathrm{C}$ NMR 199.1, $159.5,152.0,150.1,141.7,138.9,136.1,131.35,131.29,127.1,116.8,113.6,112.7,105.6,92.6,61.4$, 60.9, 56.2, 55.4, 29.4; MS m/e $596\left(\mathrm{M}^{+}\right), 568\left(\mathrm{M}^{+}-\mathrm{CO}\right), 540\left(\mathrm{M}^{+}-2 \mathrm{CO}\right), 512\left(\mathrm{M}^{+}-3 \mathrm{CO}\right), 484\left(\mathrm{M}^{+}-4 \mathrm{CO}\right)$, $456\left(\mathrm{M}^{+}-5 \mathrm{CO}\right)$; HRMS for $\mathrm{C}_{25} \mathrm{H}_{18} \mathrm{Co}_{2} \mathrm{O}_{10}\left(\mathrm{M}^{+}\right)$calcd. 595.9564, found 595.9548.

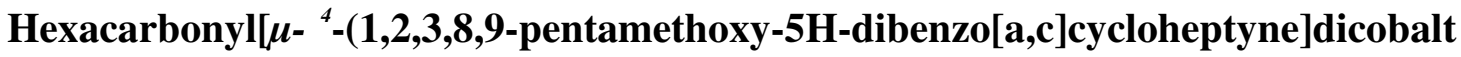

(9f).

Subjecting (8f) $(0.9250 \mathrm{~g}, 1.35 \mathrm{mmol})$ to General Procedure C (4.5 h) afforded 9f $(0.7021 \mathrm{~g}, 83 \%$ yield $)$ following flash chromatography $\left(5: 1\right.$ hexanes:Et $\left.{ }_{2} \mathrm{O}\right)$, as a dark brown solid, mp $115-117{ }^{\circ} \mathrm{C}$; $\mathrm{IR}(\mathrm{KBr}){ }_{\max }$ 2939, 2090, 2054, $1593 \mathrm{~cm}^{-1}$; ${ }^{1} \mathrm{H}$ NMR 7.30 (d, J = 8.7, 1H), 6.97 (d, J = 8.7, 1H), $6.63(\mathrm{~s}, 1 \mathrm{H}), 3.99$ (d, $\mathrm{J}=14.0,1 \mathrm{H}), 3.99(\mathrm{~s}, 3 \mathrm{H}), 3.95(\mathrm{~s}, 3 \mathrm{H}), 3.92(\mathrm{~s}, 3 \mathrm{H}), 3.81(\mathrm{~s}, 3 \mathrm{H}), 3.67(\mathrm{~d}, \mathrm{~J}=14.0,1 \mathrm{H}), 3.40(\mathrm{~s}, 3 \mathrm{H})$; ${ }^{13}$ C NMR 199.5, 153.1, 152.3, 152.0, 148.4, 142.3, 137.3, 132.2, 128.3, 126.3, 125.9, 110.6, 107.5, 107.3, 85.8, 60.7, 60.7, 60.4, 56.1, 55.8, 40.3; MS m/e $626\left(\mathrm{M}^{+}\right), 598\left(\mathrm{M}^{+}-\mathrm{CO}\right), 570\left(\mathrm{M}^{+}-2 \mathrm{CO}\right), 542\left(\mathrm{M}^{+}-\right.$ 3CO), $514\left(\mathrm{M}^{+}-4 \mathrm{CO}\right), 486\left(\mathrm{M}^{+}-5 \mathrm{CO}\right), 458\left(\mathrm{M}^{+}-6 \mathrm{CO}\right)$; HRMS for $\mathrm{C}_{26} \mathrm{H}_{20} \mathrm{Co}_{2} \mathrm{O}_{11}\left(\mathrm{M}^{+}\right)$calcd. 625.9670, found 625.9673 .

\section{Hexacarbonyl[ $\mu$ - ${ }^{4}$-(5H-dibenzo[a,c]cycloheptyne]dicobalt (9g). Subjecting 8g (0.0604 g, 0.127} mmol) to General Procedure C (16 h) afforded 9g $(0.0311,58 \%$ yield) following flash chromatography (100\% petroleum ether), as a dark brown solid, mp 130-132 ${ }^{\circ} \mathrm{C}$; IR (KBr) ${ }_{\max } 3059,2959,1688,2091$, 2052, $2022 \mathrm{~cm}^{-1} ;{ }^{1} \mathrm{H}$ NMR $7.71(\mathrm{~m}, 1 \mathrm{H}), 7.59(\mathrm{~s}, 1 \mathrm{H}), 7.40-7.47(\mathrm{~m}, 3 \mathrm{H}), 7.28-7.36(\mathrm{~m}, 3 \mathrm{H}), 4.05$ (s, $2 \mathrm{H}) ;{ }^{13} \mathrm{C}$ NMR 199.0, 140.4, 140.2, 138.5, 137.3, 133.2, 132.1, 130.4, 128.6, 128.3, 127.92, 127.86, 
127.5, 104.2, 92.0, 39.9; MS m/e $420\left(\mathrm{M}^{+}-2 \mathrm{CO}\right), 336\left(\mathrm{M}^{+}-5 \mathrm{CO}\right), 308\left(\mathrm{M}^{+}-6 \mathrm{CO}\right)$; HRMS m/e for $\mathrm{C}_{21} \mathrm{H}_{10} \mathrm{Co}_{2} \mathrm{O}_{6}$ calcd. $447.9192\left(\mathrm{M}^{+}-\mathrm{CO}\right)$, found 447.9214.

\section{Hexacarbonyl[ $\mu$ - ${ }^{4}$-(9-carbomethoxy-1,2,3-trimethoxy-5H-dibenzo[a,c]cycloheptyne $]$ dicobalt}

(9h); Subjecting (8h) (0.2304 g, $0.351 \mathrm{mmol})$ to General Procedure C (5 h) afforded 9h $(0.1831 \mathrm{~g}, 84 \%$ yield) following flash chromatography (5:1 petroleum ether: $\left.\mathrm{Et}_{2} \mathrm{O}\right)$, as a dark brown viscous oil; IR $(\mathrm{KBr})_{\text {max }} 2952,2092,2055,2023,1730 \mathrm{~cm}^{-1} ;{ }^{1} \mathrm{H}$ NMR $\quad 8.30(\mathrm{~d}, \mathrm{~J}=1.9,1 \mathrm{H}), 7.98(\mathrm{dd}, \mathrm{J}=8.2,1.9$, 1H), $7.69(\mathrm{~d}, \mathrm{~J}=8.2,1 \mathrm{H}), 6.67(\mathrm{~s}, 1 \mathrm{H}), 4.05(\mathrm{~d}, \mathrm{~J}=14.1,1 \mathrm{H}), 3.98(\mathrm{~s}, 3 \mathrm{H}), 3.95(\mathrm{~s}, 3 \mathrm{H}), 3.84(\mathrm{~s}, 3 \mathrm{H})$, $3.65(\mathrm{~d}, \mathrm{~J}=14.1,1 \mathrm{H}), 3.39(\mathrm{~s}, 3 \mathrm{H}) ;{ }^{13} \mathrm{C} \mathrm{NMR} 198.7,166.7,153.24,153.15,142.4,138.3,137.9,137.0$, $132.9,129.4,126.8,125.5,107.9,105.4,91.4,61.1,60.6,56.1,52.3,39.9 ; \mathrm{MS}$ m/e $596\left(\mathrm{M}^{+}-1 \mathrm{CO}\right), 568$ ( $\left.\mathrm{M}^{+}-2 \mathrm{CO}\right), 540\left(\mathrm{M}^{+}-3 \mathrm{CO}\right), 512\left(\mathrm{M}^{+}-4 \mathrm{CO}\right), 484\left(\mathrm{M}^{+}-5 \mathrm{CO}\right)$; ); HRMS for $\mathrm{C}_{26} \mathrm{H}_{18} \mathrm{Co}_{2} \mathrm{O}_{11}\left(\mathrm{M}^{+}-3 \mathrm{CO}\right)$ calcd. 539.9666, found 539.9683.

1,2,3,9-Tetramethoxy-5H-dibenzo[a,c]cycloheptene (17a). To a solution of 9a $(0.0782 \mathrm{~g}, 0.131$ mmol) in degassed 1,2-dichloroethane $(2 \mathrm{~mL})$ was added bis(trimethylsilyl)acetylene (62 $\mu \mathrm{L}, .274$ mmol) and triethylsilane $(0.10 \mathrm{~mL}, 0.63 \mathrm{mmol})$. The mixture was heated to $65{ }^{\circ} \mathrm{C}$ for $6 \mathrm{~h}$ and cooled to room temperature, at which point trifluoroacetic acid $(0.5 \mathrm{~mL})$ was added. After stirring for an additional $12 \mathrm{~h}$, the mixture was subjected to a conventional extractive workup. Preparative TLC (4:1 hexanes : $\left.\mathrm{Et}_{2} \mathrm{O}\right)$ afforded $\mathbf{1 7 a}(0.0396 \mathrm{~g}, 97 \%$ yield $)$ as a colorless solid, mp 102-3 ${ }^{\circ} \mathrm{C}(\mathrm{MeOH})$, Lit. $^{29}$ $102-3{ }^{\circ} \mathrm{C}(\mathrm{MeOH})$.

2,3,4,9-Tetramethoxy-5H-dibenzo[a,c]cycloheptene (17e). To a solution of 9e $(0.3492 \mathrm{~g}, 0.586$ mmol) in degassed 1,2-dichloroethane $(20 \mathrm{~mL})$ was added bis(trimethylsilyl)acetylene $(0.27 \mathrm{~mL}, 1.2$ mmol) and triethylsilane $(0.47 \mathrm{~mL}, 2.9 \mathrm{mmol})$. The mixture was heated to $65{ }^{\circ} \mathrm{C}$ for $6 \mathrm{~h}$ and cooled to room temperature, at which point trifluoroacetic acid $(1.0 \mathrm{~mL})$ was added. After stirring for an additional $12 \mathrm{~h}$, the mixture was subjected to a conventional extractive workup. Preparative TLC (10:1

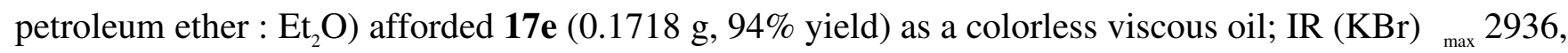
$1604 \mathrm{~cm}^{-1} ;{ }^{1} \mathrm{H}$ NMR $7.61(\mathrm{~d}, \mathrm{~J}=8.7,1 \mathrm{H}), 6.95(\mathrm{dd}, \mathrm{J}=8.7,2.6,1 \mathrm{H}), 6.85(\mathrm{~d}, \mathrm{~J}=2.6,1 \mathrm{H}), 6.82(\mathrm{~s}, 1 \mathrm{H})$, $6.57(\mathrm{~d}, \mathrm{~J}=10.0,1 \mathrm{H}), 6.26(\mathrm{~m}, 1 \mathrm{H}), 3.94(\mathrm{~s}, 3 \mathrm{H}), 3.93(\mathrm{~s}, 3 \mathrm{H}), 3.90(\mathrm{~s}, 3 \mathrm{H}), 3.89$ (s, 3H), 2.47 (v br, 
$2 \mathrm{H}) ;{ }^{13} \mathrm{C}$ NMR $158.2,151.1,148.9,141.9,137.4,134.3,132.8,132.3,130.7,129.6,128.3,113.4,112.9$, 109.1, 61.5, 60.9, 56.2, 55.3, 24.0; MS m/e $312\left(\mathrm{M}^{+}\right)$; HRMS for $\mathrm{C}_{19} \mathrm{H}_{20} \mathrm{O}_{4}\left(\mathrm{M}^{+}\right)$calcd. 312.1362, found 312.1356.

1,2,3,8,9-Pentaamethoxy-5H-dibenzo[a,c]cycloheptene (17f). To a solution of 9f $(0.0945 \mathrm{~g}, 0.151$ mmol) in degassed 1,2-dichloroethane $(2 \mathrm{~mL})$ was added bis(trimethylsilyl)acetylene $(62 \mu \mathrm{L}, 0.32$ mmol) and triethylsilane $(0.12 \mathrm{~mL}, 0.76 \mathrm{mmol})$. The mixture was heated to $65{ }^{\circ} \mathrm{C}$ for $6 \mathrm{~h}$ and cooled to room temperature, at which point trifluoroacetic acid $(0.5 \mathrm{~mL})$ was added. After stirring for an additional $12 \mathrm{~h}$, the mixture was subjected to a conventional extractive workup. Preparative TLC (7:1 petroleum ether : $\left.\mathrm{Et}_{2} \mathrm{O}\right)$ afforded $\mathbf{1 7 f}\left(0.0465 \mathrm{~g}, 90 \%\right.$ yield) as a colorless solid, mp $163-165{ }^{\circ} \mathrm{C}$; IR $(\mathrm{KBr})_{\max } 3036,2926,1573 \mathrm{~cm}^{-1} ;{ }^{1} \mathrm{H}$ NMR $7.55(\mathrm{~d}, \mathrm{~J}=8.7,1 \mathrm{H}), 6.94(\mathrm{~d}, \mathrm{~J}=8.7,1 \mathrm{H}), 6.78(\mathrm{~d}, \mathrm{~J}=10.1$, 1H), $6.58(\mathrm{~s}, 1 \mathrm{H}), 6.29(\mathrm{~m}, 1 \mathrm{H}), 3.95(\mathrm{~s}, 3 \mathrm{H}), 3.91(\mathrm{~s}, 3 \mathrm{H}), 3.90(\mathrm{~s}, 3 \mathrm{H}), 3.85(\mathrm{~s}, 3 \mathrm{H}), 3.48(\mathrm{~s}, 3 \mathrm{H}), 3.06$ $(\mathrm{dd}, \mathrm{J}=12.9,7.9,1 \mathrm{H}), 2.80(\mathrm{ddd}, \mathrm{J}=12.9,5.9,1.8,1 \mathrm{H}) ;{ }^{13} \mathrm{C}$ NMR 152.3, 150.4, 145.2, 140.7, 140.1, $132.2,131.1,128.3,127.5,124.0,123.8,109.9,105.6,61.0,60.6,60.4,55.9,55.8,33.5 ;$ MS m/e 342 $\left(\mathrm{M}^{+}\right)$; HRMS for $\mathrm{C}_{20} \mathrm{H}_{22} \mathrm{O}_{5}$ calcd. 342.1467, found 342.1475.

9-Carbomethoxy-1,2,3-trimethoxy-5H-dibenzo[a,c]cycloheptene (17h). To a solution of 9h $(0.1831 \mathrm{~g}, 0.293 \mathrm{mmol})$ in degassed 1,2-dichloroethane $(7 \mathrm{~mL})$ was added bis(trimethylsilyl)acetylene $(0.14 \mathrm{~mL}, 0.59 \mathrm{mmol})$ and triethylsilane $(0.23 \mathrm{~mL}, 0.14 \mathrm{mmol})$. The mixture was heated to $65{ }^{\circ} \mathrm{C}$ for $6 \mathrm{~h}$ and cooled to room temperature, at which point trifluoroacetic acid $(3.0 \mathrm{~mL})$ was added. After stirring for an additional $12 \mathrm{~h}$, the mixture was subjected to a conventional extractive workup. Preparative TLC (5:1 hexanes : $\left.\mathrm{Et}_{2} \mathrm{O}\right)$ afforded $\mathbf{1 7 h}\left(0.0 .0789 \mathrm{~g}, 79 \%\right.$ yield) as a colorless solid, mp 85-86 ${ }^{\circ} \mathrm{C}$; IR $(\mathrm{KBr})$ ${ }_{\max } 2950,1722,1594 \mathrm{~cm}^{-1} ;{ }^{1} \mathrm{H}$ NMR $8.03(\mathrm{~s}, 1 \mathrm{H}), 7.91(1 / 2 \mathrm{AB}$ quartet, $\mathrm{J}=8.3,1 \mathrm{H}), 7.89(1 / 2 \mathrm{AB}$ quartet, $\mathrm{J}=8.3,1 \mathrm{H}), 6.62(\mathrm{~d}, \mathrm{~J}=10.0,1 \mathrm{H}), 6.59(\mathrm{~s}, 1 \mathrm{H}), 6.29(\mathrm{~m}, 1 \mathrm{H}), 3.96(\mathrm{~s}, 3 \mathrm{H}), 3.92(\mathrm{~s}, 3 \mathrm{H}), 3.90$ (s, 3H), $3.46(\mathrm{~s}, 3 \mathrm{H}), 3.08(\mathrm{~m}, 1 \mathrm{H}), 2.75(\mathrm{~m}, 1 \mathrm{H}) ;{ }^{13} \mathrm{C}$ NMR 167.0, 153.3, 152.3, 140.8, 140.5, 139.4, $136.5,133.2,132.0,130.1,128.8,127.6,125.4,123.3,105.8,61.1,60.7,55.9,52.1,33.3 ; \mathrm{MS} \mathrm{m} / \mathrm{e} 340$ $\left(\mathrm{M}^{+}\right)$; HRMS for $\mathrm{C}_{20} \mathrm{H}_{20} \mathrm{O}_{5}\left(\mathrm{M}^{+}\right)$calcd. 340.1311, found 340.1306. 
6,7-Dihydro-3,9,10,11-tetramethoxy-5H-dibenzo[a,c]cyclohepten-5-one (18a). To a solution of alkene $17 \mathbf{a}(0.0521 \mathrm{~g}, 0.167 \mathrm{mmol})$ in THF $(7 \mathrm{~mL})$ at $0{ }^{\circ} \mathrm{C}$ was added $\mathrm{BH}_{3}-\mathrm{THF}(0.75 \mathrm{~mL}$ of a $1 \mathrm{M}$ solution). The cooling bath was removed, and the reaction stirred at room temperature for $12 \mathrm{~h}$. $\mathrm{NaOH}$ ( $1 \mathrm{~mL}$ of a $10 \%$ aqueous solution) and $\mathrm{H}_{2} \mathrm{O}_{2}(1 \mathrm{~mL}$ of a $33 \%$ aqueous solution) were added, and the mixture stirred for $4 \mathrm{~h}$, followed by warming to $40{ }^{\circ} \mathrm{C}$ for $0.5 \mathrm{~h}$. A conventional workup gave a crude alcohol which was added slowly as a solution in $\mathrm{CH}_{2} \mathrm{Cl}_{2}$ to a $-78{ }^{\circ} \mathrm{C}$ solution prepared from the addition of DMSO $(0.14 \mathrm{~mL}, 2.0 \mathrm{mmol})$ to oxalyl chloride $(86 \mu \mathrm{L}, 1.0 \mathrm{mmol})$ in $\mathrm{CH}_{2} \mathrm{Cl}_{2}(10 \mathrm{~mL})$ at $-78{ }^{\circ} \mathrm{C}$. Diisopropylethylamine $(0.70 \mathrm{~mL}, 4.0 \mathrm{mmol})$ was added and the solution allowed to come to room temperature over 6h. A conventional workup gave a residue with a 96:4 mixture of ketone regioisomers (by integration of relevant ${ }^{1} \mathrm{H}$ NMR resonances); preparative TLC (1:1 hexanes : $\left.\mathrm{Et}_{2} \mathrm{O}\right)$ afford 18a $\left(0.0440,80 \%\right.$ yield) as a colorless solid, mp $141-2{ }^{\circ} \mathrm{C}$ (hexanes), lit. $142-3{ }^{\circ} \mathrm{C}(\mathrm{MeOH}),{ }^{5 \mathrm{a}} 140.5-141{ }^{\circ} \mathrm{C},{ }^{30}$ $135-6{ }^{\circ} \mathrm{C} .{ }^{11}$

6,7-Dihydro-3,8,9,10-tetramethoxy-5H-dibenzo[a,c]cyclohepten-5-one. (18e). To a solution of alkene 17e $(0.1793 \mathrm{~g}, 0.574 \mathrm{mmol})$ in $\mathrm{THF}(20 \mathrm{~mL})$ at $0{ }^{\circ} \mathrm{C}$ was added $\mathrm{BH}_{3}-\mathrm{THF}(2.7 \mathrm{~mL}$ of a $1 \mathrm{M}$ solution). The cooling bath was removed, and the reaction stirred at room temperature for $12 \mathrm{~h}$. $\mathrm{NaOH}$ ( $3 \mathrm{~mL}$ of a $10 \%$ aqueous solution) and $\mathrm{H}_{2} \mathrm{O}_{2}$ ( $3 \mathrm{~mL}$ of a $33 \%$ aqueous solution) were added, and the mixture stirred for $4 \mathrm{~h}$, followed by warming to $40{ }^{\circ} \mathrm{C}$ for $0.5 \mathrm{~h}$. A conventional workup gave a crude alcohol which was dissolved in $\mathrm{CH}_{2} \mathrm{Cl}_{2}(20 \mathrm{~mL})$. To this solution was added PDC (0.55 g), and the mixture was stirred for $12 \mathrm{~h}$. A conventional workup followed by preparative TLC (3:1 petroleum ether:Et $\left.{ }_{2} \mathrm{O}\right)$ afforded $18 \mathrm{e}\left(0.1291 \mathrm{~g}, 68 \%\right.$ yield) as a colorless solid, mp $123-125{ }^{\circ} \mathrm{C}$; IR (KBr) ${ }_{\text {max }} 2937$, 1681, $1601 \mathrm{~cm}^{-1} ;{ }^{1} \mathrm{H}$ NMR $7.36(\mathrm{~m}, 1 \mathrm{H}), 7.14(\mathrm{~s}, 1 \mathrm{H}), 7.13$ (obscured m, 1H), 6.69 (s, 1H), $3.92(\mathrm{~s}$, 3H), $3.89(\mathrm{~s}, 3 \mathrm{H}), 3.88(\mathrm{~s}, 6 \mathrm{H}), 2.97-3.00(\mathrm{~m}, 2 \mathrm{H}), 2.92-2.94(\mathrm{~m}, 2 \mathrm{H}) ;{ }^{13} \mathrm{C}$ NMR 206.3, 158.9, 152.2, $150.1,141.6,139.7,134.4,131.4,130.5,125.4,118.8,112.1,109.0,61.3,60.8,56.0,55.4,47.3,20.4$; MS m/e $328\left(\mathrm{M}^{+}\right)$; HRMS for $\mathrm{C}_{19} \mathrm{H}_{20} \mathrm{O}_{5}\left(\mathrm{M}^{+}\right)$calcd. 328.1311, found 328.1322.

6,7-Dihydro-3,4,9,10,11-pentamethoxy-5H-dibenzo[a,c]cyclohepten-5-one (18f). To a solution of alkene $17 f(0.2175 \mathrm{~g}, 0.636 \mathrm{mmol})$ in $\mathrm{THF}(25 \mathrm{~mL})$ at $0{ }^{\circ} \mathrm{C}$ was added $\mathrm{BH}_{3}-\mathrm{THF}(3.0 \mathrm{~mL}$ of a $1 \mathrm{M}$ 
solution). The cooling bath was removed, and the reaction stirred at room temperature for $12 \mathrm{~h}$. $\mathrm{NaOH}$ ( $3 \mathrm{~mL}$ of a $10 \%$ aqueous solution) and $\mathrm{H}_{2} \mathrm{O}_{2}(3 \mathrm{~mL}$ of a $33 \%$ aqueous solution) were added, and the mixture stirred for $4 \mathrm{~h}$, followed by warming to $40{ }^{\circ} \mathrm{C}$ for $0.5 \mathrm{~h}$. A conventional workup gave a crude alcohol which was dissolved in $\mathrm{CH}_{2} \mathrm{Cl}_{2}(20 \mathrm{~mL})$. To this solution was added PDC (0.55 $\left.\mathrm{g}\right)$, and the mixture was stirred for $12 \mathrm{~h}$. A conventional workup followed by preparative TLC (3:1 petroleum ether:Et $\left.{ }_{2} \mathrm{O}\right)$ afforded $\mathbf{1 8 f}(0.1505 \mathrm{~g}, 67 \%$ yield $)$ as a colorless solid, mp $164-165{ }^{\circ} \mathrm{C}$, lit. $156-157{ }^{\circ} \mathrm{C} ;{ }^{22} \mathrm{IR}$ $(\mathrm{KBr})_{\max } 2939,1701,1598 \mathrm{~cm}^{-1} ;{ }^{1} \mathrm{H}$ NMR $7.26(\mathrm{~d}, \mathrm{~J}=8.6,1 \mathrm{H}), 7.00(\mathrm{~d}, \mathrm{~J}=8.6,1 \mathrm{H}), 6.56(\mathrm{~s}, 1 \mathrm{H})$, $3.91(\mathrm{~s}, 3 \mathrm{H}), 3.87(\mathrm{~s}, 3 \mathrm{H}), 3.86(\mathrm{~s}, 6 \mathrm{H}), 3.57(\mathrm{~s}, 3 \mathrm{H}), 2.97-3.05(\mathrm{~m}, 2 \mathrm{H}), 2.85(\mathrm{~m}, 1 \mathrm{H}), 2.59(\mathrm{~m}, 1 \mathrm{H}) ;{ }^{13} \mathrm{C}$ NMR 204.8, 152.6, 151.8, 151.7, 144.3, 141.4, 135.24, 135.21, 126.24, 126.16, 123.8, 113.0, 107.5, 62.2, 61.0, 60.8, 55.9, 55.8, 49.6, 30.1; MS m/e $354\left(\mathrm{M}^{+}\right)$; HRMS for $\mathrm{C}_{20} \mathrm{H}_{22} \mathrm{O}_{6}\left(\mathrm{M}^{+}\right)$calcd. 358.1416, found 358.1402 .

\section{6,7-Dihydro-3-carbomethoxy-9,10,11-trimethoxy-5H-dibenzo[a,c]cyclohepten-5-one (18h). To a} solution of alkene $\mathbf{1 7 h}(0.0307 \mathrm{~g}, 0.0902 \mathrm{mmol})$ in $\mathrm{THF}(5 \mathrm{~mL})$ at $0{ }^{\circ} \mathrm{C}$ was added $\mathrm{BH}_{3}-\mathrm{THF}(0.45 \mathrm{~mL}$ of a $1 \mathrm{M}$ solution). The cooling bath was removed, and the reaction stirred at room temperature for $12 \mathrm{~h}$. $\mathrm{NaOH}\left(1 \mathrm{~mL}\right.$ of a $10 \%$ aqueous solution) and $\mathrm{H}_{2} \mathrm{O}_{2}(1 \mathrm{~mL}$ of a $33 \%$ aqueous solution $)$ were added, and the mixture stirred for $4 \mathrm{~h}$, followed by warming to $40{ }^{\circ} \mathrm{C}$ for $0.5 \mathrm{~h}$. A conventional workup gave a crude alcohol which was dissolved in $\mathrm{CH}_{2} \mathrm{Cl}_{2}(10 \mathrm{~mL})$. To this solution was added PDC (0.5 g), and the mixture was stirred for $12 \mathrm{~h}$. A conventional workup followed by preparative TLC (1:1 petroleum ether:Et $\left.{ }_{2} \mathrm{O}\right)$ afforded $\mathbf{1 8 h}(0.0 .0260 \mathrm{~g}, 81 \%$ yield $)$ as a colorless solid, mp 144-145 ${ }^{\circ} \mathrm{C}$; lit. $^{6}$ 144.2-144.8 ${ }^{\circ} \mathrm{C}$.

(5R)- 6,7-Dihydro-3,9,10,11-tetramethoxy-5H-dibenzo[a,c]cyclohepten-5-ol (19a). A suspension of 3 -nitrophenylboronic acid $(0.334 \mathrm{~g}, 2.0 \mathrm{mmol})$, L-tartaric acid $(0.0300 \mathrm{~g}, 2.0 \mathrm{mmol})$ and $\mathrm{CaH}_{2}(0.168$ $\mathrm{g}, 4.0 \mathrm{mmol})$ in THF (5 mL)was heated to reflux for $1 \mathrm{~h}$. After cooling and allowing the solids to settle, the supernatant solution $(2.5 \mathrm{~mL}$, ca. $1 \mathrm{mmol})$ was added to ketone $18 \mathrm{a}(0.0634 \mathrm{~g}, 0.193 \mathrm{mmol})$. Lithium borohydride $(0.5 \mathrm{~mL}$ of a $2 \mathrm{M}$ solution, $1.0 \mathrm{mmol})$ was added over a period of $5 \mathrm{~min}$, and the solution stirred for $0.5 \mathrm{~h}$. $\mathrm{NaOH}(1 \mathrm{~mL}$ of a $10 \%$ aqueous solution) and water $(2 \mathrm{~mL})$ were added, and 
the reaction subjected to a conventional extractive workup. Preparative TLC $\left(2: 1\right.$ pet $\left.\operatorname{ether:Et}_{2} \mathrm{O}\right)$ afforded 19a $(0.0610 \mathrm{~g}, 96 \%), 95 \%$ ee (Chiralcel OD-H, $i$-PrOH-hexanes), mp 137-9 ${ }^{\circ} \mathrm{C}\left(\mathrm{CH}_{2} \mathrm{Cl}_{2}:\right.$ pet ether) []$_{\mathrm{D}}^{22} 120^{\circ}(\mathrm{c} 0.0144) ;{ }^{1} \mathrm{H}$ NMR $\left(\mathrm{DMSO}-\mathrm{d}^{6}\right) \quad 7.24(\mathrm{~d}, \mathrm{~J}=8.4,1 \mathrm{H}), 7.17(\mathrm{~d}, \mathrm{~J}=2.8,1 \mathrm{H}), 6.85(\mathrm{dd}$, $\mathrm{J}=84,2.8,1 \mathrm{H}), 6.75(\mathrm{~s}, 1 \mathrm{H}), 5.23(\mathrm{~d}, \mathrm{~J}=4.7,1 \mathrm{H}), 4.27(\mathrm{~m}, 1 \mathrm{H}), 3.82(\mathrm{~s}, 3 \mathrm{H}), 3.80(\mathrm{~s}, 3 \mathrm{H}), 3.75(\mathrm{~s}, 3 \mathrm{H})$, $3.48(\mathrm{~s}, 3 \mathrm{H}), 2.32-2.45(\mathrm{~m}, 2 \mathrm{H}), 2.06(\mathrm{~m}, 1 \mathrm{H}), 1.73(\mathrm{~m}, 1 \mathrm{H}) ;{ }^{13} \mathrm{C}$ NMR (DMSO-d $\left.{ }^{6}\right)$ 158.3, 151.9, 150.2, $144.5,140.4,135.2,130.3,124.9,124.0,111.2,108.7,107.9,68.1,60.5,60.4,55.8,54.9,41.3,29.9$; HRMS m/e for $\mathrm{C}_{19} \mathrm{H}_{22} \mathrm{O}_{5}$ calcd. $330.1467\left(\mathrm{M}^{+}\right)$, found 330.1479 .

(5R)- 6,7-Dihydro-3,8,9,10-tetramethoxy-5H-dibenzo[a,c]cyclohepten-5-ol (19e). A suspension of 3-nitrophenylboronic acid (0.334 g, $2.0 \mathrm{mmol})$, L-tartaric acid (0.0300 $2.0 \mathrm{mmol})$ and $\mathrm{CaH}_{2}(0.168 \mathrm{~g}$, $4.0 \mathrm{mmol})$ in THF (5 mL)was heated to reflux for $1 \mathrm{~h}$. After cooling and allowing the solids to settle, the supernatant solution $(2.5 \mathrm{~mL}$, ca. $1 \mathrm{mmol})$ was added to ketone $\mathbf{1 8 e}(0.0217 \mathrm{~g}, 0.0661 \mathrm{mmol})$. Lithium borohydride $(0.2 \mathrm{~mL}$ of a $2 \mathrm{M}$ solution, $0.4 \mathrm{mmol})$ was added over a period of $1.5 \mathrm{~h}$, and the solution stirred for $0.5 \mathrm{~h}$. $\mathrm{NaOH}(1 \mathrm{~mL}$ of a $10 \%$ aqueous solution) and water $(2 \mathrm{~mL})$ were added, and the reaction subjected to a conventional extractive workup. Preparative TLC (1:1 petroleum ether:Et $\left.{ }_{2} \mathrm{O}\right)$ afforded 19e $(0.0213 \mathrm{~g}, 98 \%), 98 \%$ ee (Chiralcel AS-H, 10\% $i$-PrOH-hexanes) as a viscous oil, [ $]_{\mathrm{D}}^{22}$ $102^{\circ}$ (c 0.533); IR (KBr) ${ }_{\text {max }} 3500 \mathrm{br}, 2936,1646 \mathrm{~cm}^{-1} ;{ }^{1} \mathrm{H}$ NMR (DMSO-d $\left.)_{6}\right) 7.30(\mathrm{~d}, \mathrm{~J}=8.5,1 \mathrm{H}), 7.18$ $(\mathrm{d}, \mathrm{J}=2.5,1 \mathrm{H}), 6.91(\mathrm{dd}, \mathrm{J}=8.5,2.5,1 \mathrm{H}), 5.25(\mathrm{~d}, \mathrm{~J}=4.5,1 \mathrm{H}), 3.83(\mathrm{~s}, 3 \mathrm{H}), 3.81(\mathrm{~s}, 3 \mathrm{H}), 3.79(\mathrm{~s}, 3 \mathrm{H})$, $3.78(\mathrm{~s}, 3 \mathrm{H}), 2.86(\mathrm{~m}, 1 \mathrm{H}), 2.38(\mathrm{~m}, 3 \mathrm{H}), 1.75-1.86(\mathrm{~m}, 2 \mathrm{H}) ;{ }^{13} \mathrm{C} \mathrm{NMR}\left(\mathrm{CDCl}_{3}\right)$ 159.4, 151.7, 150.8, 143.2, 141.3, 135.4, 130.3, 129.0, 125.0, 112.5, 108.7 (br), 107.9, 70.7 (br), 61.6, 61.0, 55.1, 55.4, 41.5, 21.5; MS m/e $330\left(\mathrm{M}^{+}\right)$; HRMS for $\mathrm{C}_{19} \mathrm{H}_{22} \mathrm{O}_{5}\left(\mathrm{M}^{+}\right)$calcd. 330.1467, found 330.1481.

(5S)- 5-Azido-6,7-dihydro-3,9,10,11-tetramethoxy-5H-dibenzo[a,c]cycloheptene (20a). To a suspension of alcohol 19a $(0.0583 \mathrm{~g}, 0.176 \mathrm{mmol}), \mathrm{Zn}\left(\mathrm{N}_{3}\right)_{2}$-(pyridine $)_{2}(0.0810 \mathrm{~g}, 0.264 \mathrm{mmol})$, and triphenylphosphine $(0.185 \mathrm{~g}, 0.704 \mathrm{mmol})$ in toluene $(2 \mathrm{~mL})$ was added diisopropyl azodicarboxylate $(0.14 \mathrm{~mL}, 0.70 \mathrm{mmol})$ in a dropwise fashion. After stirring for $4 \mathrm{~h}$, the mixture was filtered through a plug of silica gel, and concentrated under reduced pressure. Preparative TLC (4:1 hexanes: $\left.\mathrm{Et}_{2} \mathrm{O}\right)$ afforded 20a contaminated by $10 \%$ of alkene $17 \mathbf{a}(0.0440 \mathrm{~g}, 64 \%$ of $\mathbf{2 0 a}, 7 \%$ of 17a). Repeated 
preparative TLC (10:1 hexanes : EtOAc) afforded pure 20a as a viscous oil, []$_{\mathrm{D}}^{22} 110^{\circ}$ (c 0.0100$)(93 \%$ ee material as evaluated on 2), IR (KBr) ${ }_{\max } 2936,2013 \mathrm{~cm}^{-1} ;{ }^{1} \mathrm{H}$ NMR (major atropisomer, 91\%) $7.42(\mathrm{~d}, \mathrm{~J}=8.5,1 \mathrm{H}), 7.13(\mathrm{~d}, \mathrm{~J}=2.5,1 \mathrm{H}), 6.92(\mathrm{dd}, \mathrm{J}=8.5,2.5,1 \mathrm{H}), 6.60(\mathrm{~s}, 1 \mathrm{H}), 4.44(\mathrm{dd}, \mathrm{J}=11.5$, 7.0, 1H), $3.92(\mathrm{~s}, 6 \mathrm{H}), 3.90(\mathrm{~s}, 3 \mathrm{H}), 3.65(\mathrm{~s}, 3 \mathrm{H}), 2.45-2.60(\mathrm{~m}, 2 \mathrm{H}), 2.33(\mathrm{~m}, 1 \mathrm{H}), 2.00(\mathrm{~m}, 1 \mathrm{H})$; resonances from the minor atropisomer $(9 \%)$ could be observed at $7.42(\mathrm{~d}, \mathrm{~J}=8.8,1 \mathrm{H}), 6.97(\mathrm{dd}, \mathrm{J}=$ 8.8, 2.6, 1H), $6.81(\mathrm{~d}, \mathrm{~J}=2.6,1 \mathrm{H}), 6.58(\mathrm{~s}, 1 \mathrm{H}), 4.71(\mathrm{~d}, \mathrm{~J}=6.8,1 \mathrm{H}), 3.93(\mathrm{~s}, 3 \mathrm{H}), 3.88(\mathrm{~s}, 3 \mathrm{H}), 3.62(\mathrm{~s}$, $3 \mathrm{H}) ;{ }^{13} \mathrm{C}$ NMR 159.0, 152.6, 150.9, 141.2, 138.6, 134.7, 131.7, 126.0, 124.3, 112.5, 109.2, 107.6, 61.1, 61.0, 60.8, 56.0, 55.3, 38.9, 30.4; MS m/e $355\left(\mathrm{M}^{+}\right)$; HRMS m/e for $\mathrm{C}_{19} \mathrm{H}_{21} \mathrm{~N}_{3} \mathrm{O}_{4}$ calcd. $355.1532\left(\mathrm{M}^{+}\right)$, found 355.1541 .

(S)- N-Acetyl-O-methyl-colchicinol, (NSC 51046) (2). To a solution containing azide 20a with $10 \%$ alkene 17a $\left(0.0186 \mathrm{~g}, 48.1 \mu \mathrm{mol}\right.$ 20a) in $100 \% \mathrm{EtOH}$ saturated with $\mathrm{H}_{2}$ was added Lindlar catalyst $(0.0068 \mathrm{~g})$. The mixture was stirred under $\mathrm{H}_{2}$ for $20 \mathrm{~h}$, filtered through Celite ${ }^{\circledR}$ and concentrated under reduced pressure. The residue was dissolved in $\mathrm{CH}_{2} \mathrm{Cl}_{2}$, cooled to $0{ }^{\circ} \mathrm{C}$, and acetic anhydride $(0.2 \mathrm{~mL})$ and pyridine $(0.2 \mathrm{~mL})$ were added. The mixture was allowed to stir $12 \mathrm{~h}$ with gradual warming to room temperature. Concentration under reduced pressure, followed by preparative TLC (19:1 $\mathrm{CH}_{2} \mathrm{Cl}_{2}$ : $\mathrm{MeOH})$ afforded 2 (0.0157 g, 88\% yield), which was spectroscopically identical with authentic material, ${ }^{5,11} 93 \%$ ee (Chiralcel OD-H, 10\% i-PrOH-hexanes). A single recrystallization afforded 2 of $>$ $99 \%$ ee, mp 203-4 ${ }^{\circ} \mathrm{C}\left(\mathrm{CH}_{2} \mathrm{Cl}_{2} /\right.$ hexanes $)$; lit. ${ }^{4 \mathrm{~b}} 204-5{ }^{\circ} \mathrm{C}\left(\mathrm{CH}_{2} \mathrm{Cl}_{2} /\right.$ hexanes $) ;[]_{\mathrm{D}}{ }^{24}-64^{\circ}$ (c $\left.0.0056, \mathrm{CHCl}_{3}\right)$; lit. $^{4 \mathrm{~b}}[]_{\mathrm{D}}^{20}-65^{\mathrm{o}}\left(\mathrm{c} 0.46, \mathrm{CHCl}_{3}\right) ;$ lit. $^{4 \mathrm{a}}[]_{\mathrm{D}}^{20}-64.9^{\circ}\left(\mathrm{c} 1.03 \%, \mathrm{CHCl}_{3}\right) ;{ }^{1} \mathrm{H}$ NMR $\left(\mathrm{DMSO}-\mathrm{d}^{6}\right) \quad 8.34(\mathrm{~d}, \mathrm{~J}=$ 8.6, 1H), $7.25(\mathrm{~d}, \mathrm{~J}=8.4,1 \mathrm{H}), 6.91(\mathrm{~d}, \mathrm{~J}=2.6,1 \mathrm{H}), 6.87(\mathrm{dd}, \mathrm{J}=8.4,2.6,1 \mathrm{H}), 6.76(\mathrm{~s}, 1 \mathrm{H}), 4.52(\mathrm{~m}$, 1H), $3.83(\mathrm{~s}, 3 \mathrm{H}), 3.79(\mathrm{~s}, 3 \mathrm{H}), 3.78(\mathrm{~s}, 3 \mathrm{H}), 3.47(\mathrm{~s}, 3 \mathrm{H}), 2.48(\mathrm{~m}, 1 \mathrm{H}$, obscured), $2.15(\mathrm{~m}, 1 \mathrm{H}), 2.07$ $(\mathrm{m}, 1 \mathrm{H}), 1.88(\mathrm{~s}, 3 \mathrm{H}), 1.85(\mathrm{~m}, 1 \mathrm{H}) ;{ }^{13} \mathrm{C}$ NMR $\left(\mathrm{DMSO}^{\mathrm{d}} \mathrm{d}^{6}\right)$ 168.2, 158.3, 152.1, 150.3, 141.8, 140.5, $134.7,130.5,126.1,124.3,110.7,109.4,108.1,60.50,60.45,55.8,54.9,48.1,30.1,22.6$.

(5S)- 5-Azido-6,7-dihydro-3,8,9,10-tetramethoxy-5H-dibenzo[a,c]cycloheptene (20e). To a suspension of $19 \mathrm{e}(0.0346 \mathrm{~g}, 0.105 \mathrm{mmol}), \mathrm{Zn}\left(\mathrm{N}_{3}\right)_{2}-2$ pyridine $(0.0484 \mathrm{~g}, 0.157 \mathrm{mmol})$, and triphenylphosphine $(0.1099 \mathrm{~g}, 0.419 \mathrm{mmol})$ in toluene $(3 \mathrm{~mL})$ was added diisopropyl azodicarboxylate 
$(81 \mu \mathrm{L}, 0.42 \mathrm{mmol})$ in a dropwise fashion. After stirring for $4 \mathrm{~h}$, the mixture was filtered through a plug of silica gel, and concentrated under reduced pressure. Preparative TLC (4:1 hexanes:EtOAc) afforded 20e $(0.0286 \mathrm{~g}, 77 \%$ yield $)$ as a viscous oil; []$_{\mathrm{D}}{ }^{24}-126^{\circ}\left(\mathrm{c} 0.663, \mathrm{CHCl}_{3}\right)(95 \%$ ee material as evaluated

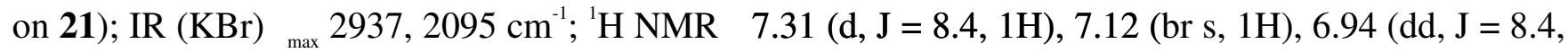
2.7, 1H), $6.70(\mathrm{~s}, 1 \mathrm{H}), 4.46(\mathrm{~m}, 1 \mathrm{H}), 3.94(\mathrm{~s}, 3 \mathrm{H}), 3.91(\mathrm{~s}, 3 \mathrm{H}), 3.905(\mathrm{~s}, 3 \mathrm{H}), 3.900$ (s, 3H), 3.01 (br m, 1H), $2.55(\mathrm{~m}, 1 \mathrm{H}), 2.06(\mathrm{~m}, 2 \mathrm{H}) ;{ }^{13} \mathrm{C}$ NMR (DMSO-d $)$ 158.8, 151.6, 150.3, 141.0, 138.0, 134.7, 130.7, 129.9, 123.4, 112.8, 110.6 (br), 108.2, 61.4, 61.2, 60.4, 55.8, 55.2, 38.1, 21.3; MS m/e 355 (M ${ }^{+}$; HRMS for $\mathrm{C}_{19} \mathrm{H}_{21} \mathrm{~N}_{3} \mathrm{O}_{4}\left(\mathrm{M}^{+}\right)$calcd. 355.1532, found 355.1537.

(5S)- 5-Acetamido-6,7-dihydro-3,8,9,10-tetramethoxy-5H-dibenzo[a,c]cycloheptene (21).To a solution containing azide 20e with $(0.0286 \mathrm{~g}, 80.5 \mu \mathrm{mol})$ in $100 \% \mathrm{EtOH}(10 \mathrm{~mL})$ saturated with $\mathrm{H}_{2}$ was added Lindlar catalyst $(0.0109 \mathrm{~g})$. The mixture was stirred under $\mathrm{H}_{2}$ for $20 \mathrm{~h}$, filtered through Celite ${ }^{\circledR}$ and concentrated under reduced pressure. The residue was dissolved in $\mathrm{CH}_{2} \mathrm{Cl}_{2}(10 \mathrm{~mL})$, cooled to $0{ }^{\circ} \mathrm{C}$, and acetic anhydride $(0.2 \mathrm{~mL})$ and pyridine $(0.2 \mathrm{~mL})$ were added. The mixture was allowed to stir $12 \mathrm{~h}$ with gradual warming to room temperature. Concentration under reduced pressure, followed by preparative TLC (19:1 $\left.\mathrm{CH}_{2} \mathrm{Cl}_{2}: \mathrm{MeOH}\right)$ afforded 21 (0.0237 g, 79\% yield) 95\% ee (Chiralcel OD-H, 10\% $i$-PrOH-hexanes). A single recrystallization afforded $\mathbf{2 1}$ of $>99 \%$ ee, as colorless crystals, mp $186-188{ }^{\circ} \mathrm{C}\left(\mathrm{Et}_{2} \mathrm{O} /\right.$ hexanes $) ;[]_{\mathrm{D}}^{22}-52.4^{\circ}\left(\mathrm{c} 0.783, \mathrm{CHCl}_{3}\right)$; IR $(\mathrm{KBr}){ }_{\max } 3288 \mathrm{br}, 2936,1664 \mathrm{~cm}^{-1} ;{ }^{1} \mathrm{H}$ NMR (DMSO-d $\left.)_{6}\right) 8.41(\mathrm{~d}, \mathrm{~J}=8.4,1 \mathrm{H}), 7.32(\mathrm{~d}, \mathrm{~J}=8.1,1 \mathrm{H}), 6.88-6.94(\mathrm{~m}, 2 \mathrm{H}), 6.79(\mathrm{~s}, 1 \mathrm{H}), 4.51(\mathrm{~m}$, 1H), 3.85 (s, 3H), $3.79(\mathrm{~s}, 6 \mathrm{H}), 3.78(\mathrm{~s}, 3 \mathrm{H}), 2.91(\mathrm{dd}, \mathrm{J}=13.2,6.0,1 \mathrm{H}), 2.16(\mathrm{~m}, 1 \mathrm{H}), 1.95(\mathrm{~m}, 1 \mathrm{H})$, 1.89 (s, 3H), $1.78(\mathrm{~m}, 1 \mathrm{H}) ;{ }^{13} \mathrm{C}$ NMR $\left(\mathrm{DMSO}_{-} \mathrm{d}_{6}\right) 168.6,158.9,151.6,150.4,141.4,140.9,135.3,130.9$, $129.3,123.7,111.3,110.1,108.3,61.5,60.4,55.9,55.1,48.4,22.7,21.9 ;\left(\right.$ a resonance at 40.0 in $\mathrm{CDCl}_{3}$ is obscured in DMSO-d $\mathrm{d}_{6}$; MS m/e $371\left(\mathrm{M}^{+}\right)$; HRMS for $\mathrm{C}_{21} \mathrm{H}_{25} \mathrm{NO}_{5}\left(\mathrm{M}^{+}\right)$calcd. 371.1733, found 371.1740.

Acknowledgements. The authors are grateful to NSERC (Canada), the Canada Foundation for Innovation (CFI), and the Ontario Innovation Trust (OIT) for support of this research. 
Supporting Information Available. ${ }^{1} \mathrm{H}$ and ${ }^{13} \mathrm{C}$ spectra for all new compounds. This material is available free of charge via the Internet at http://pubs.acs.org.

\section{References}

(1) (a) Pérez-Ramírez, B.; Gorbunoff, M. J.; Timasheff, S. N. Biochemistry 1998, 37, 1646-1661. (b) Guan, J.; Zhu, X.; Brossi, A.; Tachibana, Y.; Bastow, K. F.; Verdier-Pinard, P.; Hamel, E.; McPhail, A. T.; Lee, K. Coll. Czech. Chem. Commun. 1999, 64, 217-228. (c) Büttner, F.; Bergemann, S.; Guénard, D.; Gust, R.; Seitz, G.; Thoret, S. Bioorg. Med. Chem. 2005, 13, 3497-3511. (d) Nakagawa-Goto, K.; Jung, M. K.; Hamel, E.; W. C.-C.; Bastow, K. F.; Brossi, A.; Ohta, S.; Lee, K.-H. Heterocycles 2005, $65,541-550$

(2) (a) Tojo, E.; Abu Zarga, M. H.; Freyer, A. J.; Shamma, M. J. Nat. Prod. 1989, 52, 1163-1166. (b) Al-Tel, T. H.; Abu Zarga, M. H.; Sabri, S. S.; Freyer, A. J.; Shamma, M. J. Nat. Prod. 1990, 53, 623629. (c) Abu Zarga, M. H.; Sabri, S.; Al-Tel, T. H.; Atta-ur-Rahman; Shah, Z.; Feroz, M. J. Nat. Prod. 1991, 54, 936-940. (d) see also: Yusupov, M. K.; Sadykov, A. S. J. Gen. Chem. USSR (Engl. Transl.) 1964, 34, 1686-1688; Zh. Obshch. Khim. 1964, 34, 1677-1680. (e) Baudoin, O.; Guéritte, R. In Studies in Natural Products Chemistry; Atta-ur-Rahman, Ed.; Elsevier:Amsterdam, 2003; Vol. 29, pp 355-417.

(3) Banwell, M .G.; Fam, M.-A.; Gable, R. W.; Hamel, E. J. Chem. Soc., Chem. Commun. 1994, 2647-2649.

(4) (a) Iorio, M. A. Heterocycles 1984, 22, 2207-2211. (b) Brecht, R.; Haenel, F.; Seitz, G. Liebigs Ann./Receuil 1997, 2275-2279. (c) Diler, U.; Franz, B.; Roettele, H.; Schroeder, G.; Herges, R. J. Prakt. Chem./Chem.-Zeit. 1998, 340, 468-471. (d) Bergemann, S.; Brecht, R.; Büttner, F.; Guénard, D.; Gust, R.; Seitz, G.; Stubbs, M. T.; Thoret, S. Bioorg. Med. Chem. 2003, 11, 1269-1281. 
(5) (a) Cook, J. W.; Jack, J.; Loudon, J. D.; Buchanan, G. L.; Macmillan, J. J. Chem. Soc. 1951, 13971403. (b) Sawyer, J. S.; Macdonald, T. L. Tetrahedron Lett. 1988, 29, 4839-4842. (c) Boyé, O.; Brossi, A.; Yeh, H. J. C.; Hamel, E.; Wegrzynski, B.; Toome, V. Can. J. Chem. 1992, 70, 1237-1249.

(6) Vorogushin, A. V.; Predeus, A. V.; Wulff, W. D.; Hansen, H.-J. J. Org. Chem. 2003, 68, 58265831.

(7) (a) Boyer, F.-D.; Hanna, I. Org. Lett. 2009, 9, 715-718; (b) Boyer, F.-D.; Hanna, I. Eur. J. Org. Chem. 2008, 4938-4948; (c) Boer, F.-D.; Dubois, J.; Thoret, S.; Dau, M.-E. T. H.; Hanna, I. Bioorg. Chem. 2010, 38, 149-158.

(8) (a) Wu, T. R.; Chong, J. M. Org. Lett. 2006, 8, 15-18; (b) Besong, G.; Jarowicki, K.; Kocienski, P. J.; Sliwinski, E.; Boyle, F. T. Org. Biomol. Chem., 2006, 4 , 2193-2207; (c) Broady, S. D.; Golden, M. D.; Leonard, J.; Muir, J. C.; Maudet, M. Tetrahedron Lett. 2007, 48, 4627-4630.

(9) Leblanc, M.; Fagnou, K. Org. Lett. 2005, 7, 2849-2852.

(10) Besong, G.; Billen, D.; Dager, I.; Kocienski, P.; Sliwinski, E.; Tai, L. R.; Boyle, F. T. Tetrahedron 2008, 64, 4700-4710.

(11) Seganish, W. M.; DeShong, P. Org. Lett. 2006, 8, 3951-3954.

(12) (a) Green, J. R. Eur. J. Org. Chem., 2008, 6053-6062. (b) Amiralaei, S.; Green, J. R. Chem. Commun. 2008, 4971-4973. (c) Ding, Y.; Green, J. R. Synlett 2005, 271-274. (d) Green, J. R. Synlett, 2001, 353-356. (e) Lu, Y.; Green, J. R. Synlett 2001, 243-247. (f) Patel, M. M.; Green, J. R. Chem. Commun. 1999, 509-510. (g) Green, J. R. Chem. Commun. 1998, 1751-1752.

(13) (a) Schreiber, S. L.; Sammakia, T.; Crowe, W. E. J. Am. Chem. Soc. 1986, 108, 3128-3130. (b) Iwasawa, N.; Satoh, H. J. Am. Chem. Soc. 1999, 121, 7951-7952. (c) Tanino, K.; Shimizu, T.; Miyama, M.; Kuwajima, I. J. Am. Chem. Soc. 2000, 122, 6116-6117. (d) Iwasawa, N.; Sakurada, F.; Iwamoto, 
M. Org. Lett. 2000, 2, 871-873. (e) Tanino, K.; Kondo, F.; Shimizu, T.; Miyashita, M. Org. Lett. 2002, 4, 2217-2219. (f) Carbery, D. R.; Reignier, S.; Myatt, J. W.; Miller, N. D.; Harrity, J. P. A. Angew. Chem. Int. Ed . 2002, 41, 2584-2587. (f ) Tanino, K.; Onuki, K.; Asano, K.; Miyashita, M.; Nakamura, T.; Takahashi, Y.; Kuwajima, I. J. Am. Chem. Soc. 2003, 125, 1498-1500. (g) Young, D. G. J.; Burlison, J. A.; Peters, U. J. Org. Chem. 2003, 68, 3494-3497. (h) Golovko, L.J.; Hope-Weeks, M. J.; Mays, M. J.; McPartlin, M.; Sloan, A. M.; Woods, A. D. New. J. Chem. 2004, 28, 527-534. (i) Iwasawa, N.; Inaba, K.; Nakayama, S.; Aoki, M. Angew. Chem. Int. Ed. 2005 ,44, 7447-7450. (j) Olier, C.; Gastaldi, S.; Christie, S. D. R.; Bertrand, M. P. Synlett 2007, 423-426. (k) Kennichi; Takaya, J.; Iwasawa, N. Chem. Lett. 2007, 36, 474-475. (1) For the corresponding ethers, see: Baba, T.; Huang, G.; Isobe, M. Tetrahedron 2003, 59, 6851-6872, and references therein. (m) For the corresponding amides, see: Closser, K. D.; Quintal, M. M.; Shea, K. M. J. Org. Chem. 2007, 74, 2680-2688.

(14) Went, M. J. Adv. Organomet. Chem. 1997, 41, 69-125.

(15) Kuhn, O.; Rau, D.; Mayr, H. J. Am. Chem. Soc. 1998, 120, 900-907.

(16) For a preliminary report, see: Djurdjevic, S.; Green, J. R. Org. Lett. 2007, 9, 5505-5508.

(17) Mamane, V., Hannen, P.; Fürstner, A. Chem.-Eur. J. 2004, 10 , 4556-4575.

(18) Kraszkiewicz, L.; Sosnowski, M.; Skulski, L. Synthesis 2006, 1195-1199.

(19) O’Brien, C. J.; Kantchev, E. A. B.; Valente, C.; Hadei, N.; Chass, G. A.; Lough, A.; Hopkinson, A. C.; Organ, M. G. Chem.--Eur. J. 2006, 12, 4743-4748.

(20) For other reductive decomplexation reactions, see: (a) Hosokawa, S.; Isobe, M. Tetrahedron Lett. 1998, 39, 2609-2612. (b) Takai, S.; Ploypradith, P.; Hamajima, A.; Kira, K.; Isobe, M. Synlett 2002, 588-592. (c) Isobe, M.; Yenjai, C.;Tanaka, S. Synlett 1994, 916-918. 
(21) Kira, K.; Tanda, H.; Hamajima, A.; Baba, T.; Takai, S.; Isobe, M. Tetrahedron 2002, 58, 64856492.

(22) Brecht, R.; Seitz, G.; Guenard, D.; Thoret, S. Biiorg. Med. Chem. 2000, 8, 557-562.

(23) (a) Suri, J. T.; Vu, T.; Hernandez, A.; Congdon, J.; Singaram, B. Tetrahedron Lett. 2002, 43, 3649-3652. (b) Cordes, D. B.; Nguyen, T. M.; Kwong, T. J.; Suri, J. T.; Luibrand, R. T.; Singaram, B. Eur. J. Org. Chem. 2005, 5289-5295.

(24) Viaud, M. C.; Rollin, P. Synthesis 1990, 130-132.

(25) Rapid addition of $\mathrm{LiBH}_{4}$ afforded $19 \mathrm{~b}$ in ca. $50 \%$ ee.

(26) Still, W. C.; M. Kahn, M.; Mitra, A. J. Org. Chem. 1978, 43, 2923-2925.

(27) Bianchi, D. A.; Cipulli, M. A.; Kaufman, T. S. Eur. J. Org. Chem. 2003, 4731-4736,

(28) Kessar, S. V.; Gupta, V. P.; Balakrishnan, P.; Sawal, K. K.; Mohammad, T.; Dutt, M. J. Org. Chem. 2003, 53, 1708-1713.

(29) Boye, O.; Itoh, Y.; Brossi, A. Helv. Chim. Acta 1989, 72, 1690-1696.

(30) Rapoport, H., Williams, A. R., Cisney, M. E. J. Am. Chem. Soc. 1950, 72, 3324-3325. 